\title{
Symmetric Monge-Kantorovich problems and polar decompositions of vector fields
}

\author{
Nassif Ghoussoub* \\ Department of Mathematics \\ University of British Columbia \\ Vancouver BC Canada V6T 1Z2 \\ nassif@math.ubc.ca
}

\author{
Abbas Moameni ${ }^{\dagger}$ \\ Department of Mathematics and Computer Science \\ University of Lethbridge \\ Lethbridge, AB Canada T1K 3M4 \\ abbas.momeni@uleth.ca
}

February 10, 2013, Revised September 5, 2013

\begin{abstract}
For any given integer $N \geq 2$, we show that every bounded measurable vector field from a bounded domain $\Omega$ into $\mathbb{R}^{d}$ is $N$-cyclically monotone up to a measure preserving $N$-involution. The proof involves the solution of a multidimensional symmetric Monge-Kantorovich problem, which we first study in the case of a general cost function on a product domain $\Omega^{N}$. The polar decomposition described above corresponds to a special cost function derived from the vector field in question (actually $N-1 \mathrm{of}$ them). In this case, we show that the supremum over all probability measures on $\Omega^{N}$ which are invariant under cyclic permutations and with a given first marginal $\mu$, is attained on a probability measure that is supported on the graph of a function of the form $x \rightarrow\left(x, S x, S^{2} x, \ldots, S^{N-1} x\right)$, where $S$ is a $\mu$-measure preserving transformation on $\Omega$ such that $S^{N}=I$ a.e. The proof exploits a remarkable duality between such involutions and those Hamiltonians that are $N$-cyclically antisymmetric.
\end{abstract}

\section{Introduction}

Given Borel probability measures $\mu_{1}, \mu_{2}, \ldots, \mu_{N}$ on a domain $\Omega$ of $\mathbb{R}^{d}$ and a bounded above upper semicontinuous cost function $c: \Omega^{N} \rightarrow \mathbb{R} \cup\{-\infty\}$, the multi-marginal version of the Monge-Kantorovich problem consists of maximizing

$$
\int_{\Omega^{N}} c\left(x_{1}, x_{2}, \ldots, x_{N}\right) d \pi
$$

among all probability measures $\pi$ on $\Omega^{N}$ whose i-th marginal is equal to $\mu_{i}$ for each $i=1, \ldots, N$. We shall use the notation

$$
\operatorname{MK}\left(c ; \mu_{1}, \ldots, \mu_{N}\right)=\sup \left\{\int_{\Omega^{N}} c\left(x_{1}, x_{2}, \ldots, x_{N}\right) d \pi ; \pi \in \mathcal{P}\left(\Omega^{N}\right), \quad \operatorname{proj}_{i} \pi=\mu_{i} \text { for } i=1, \ldots, N\right\} .
$$

In this paper we are concerned with the following symmetric counterpart of the Monge-Kantorovich problem:

$$
\operatorname{MK}_{\text {sym }}(c, \mu)=\sup \left\{\int_{\Omega^{N}} c\left(x_{1}, x_{2}, \ldots, x_{N}\right) d \pi ; \pi \in \mathcal{P}_{\text {sym }}\left(\Omega^{N}, \mu\right)\right\}
$$

where $\mathcal{P}_{\text {sym }}\left(\Omega^{N}, \mu\right)$ denotes the set of all probability measures on $\Omega^{N}$, which are invariant under the cyclical permutation

$$
\sigma\left(x_{1}, x_{2}, \ldots, x_{N}\right)=\left(x_{2}, x_{3}, \ldots, x_{N}, x_{1}\right) .
$$

\footnotetext{
* Partially supported by a grant from the Natural Sciences and Engineering Research Council of Canada.

$\dagger$ Research supported by a grant from the Natural Sciences and Engineering Research Council of Canada.
} 
and whose marginals are -necessarily- equal to the same probability measure $\mu$ on $\Omega$. In other words, $\pi \in \mathcal{P}_{\text {sym }}\left(\Omega^{N}, \mu\right)$ if

$$
\int_{\Omega^{N}} f\left(x_{1}, x_{2}, \ldots, x_{N}\right) d \pi=\int_{\Omega^{N}} f\left(\sigma\left(x_{1}, x_{2}, \ldots, x_{N}\right)\right) d \pi \text { for every } f \in C\left(\Omega^{N}\right),
$$

and for every $i=1, \ldots, N$,

$$
\int_{\Omega^{N}} f\left(x_{i}\right) d \pi=\int_{\Omega} f\left(x_{i}\right) d \mu \text { for every } f \in C(\Omega) .
$$

Standard results show that there exists $\pi_{0} \in \mathcal{P}_{\text {sym }}\left(\Omega^{N}, \mu\right)$ where the supremum above is attained. In this paper, we are interested in an important class of cost functions $c$, where the optimal measure $\pi_{0}$ is necessarily supported on the graph of a function of the form $x \rightarrow\left(x, S x, S^{2} x, \ldots, S^{N-1} x\right)$, where $S$ is a $\mu$-measure preserving transformation on $\Omega$ such that $S^{N}=I$ a.e.

If $c$ is finite, then one can extend the original approach of Kantorovich to the multi-marginal and cyclically symmetric case to show that (2) is dual to the following minimization problem

$$
\operatorname{DK}_{\mathrm{sym}}^{1}(c, \mu):=\inf \left\{N \int_{\Omega} u(x) d \mu ; u: \Omega \rightarrow \mathbb{R} \text { and } \sum_{j=1}^{N} u\left(x_{j}\right) \geq \frac{1}{N} \sum_{i=0}^{N-1} c\left(\sigma^{i}\left(x_{1}, \ldots, x_{N}\right)\right)\right\} .
$$

In this paper, we introduce a new dual problem involving the class $\mathcal{H}_{N}(\Omega)$ of all $N$-cyclically antisymmetric Hamiltonians on $\Omega^{N}$, that is

$$
\mathcal{H}_{N}(\Omega)=\left\{H \in C\left(\Omega^{N} ; \mathbb{R}\right) ; \sum_{i=0}^{N-1} H\left(\sigma^{i}(\mathbf{x})\right)=0 \text { for all } \mathbf{x} \in \Omega^{N}\right\} .
$$

For $H \in \mathcal{H}_{N}(\Omega)$, let $\ell_{H}^{c}$ be the "c-Legendre transform" of $H$ with respect to the last $(N-1)$ variables, i.e.,

$$
\ell_{H}^{c}(x)=\sup \left\{c\left(x, x_{2}, \ldots, x_{N}\right)-H\left(x, x_{2}, \ldots, x_{N}\right) ;\left(x_{2}, \ldots, x_{N}\right) \in \Omega^{N-1}\right\},
$$

and consider the problem

$$
\mathrm{DK}_{\mathrm{sym}}^{2}(c, \mu):=\inf \left\{\int_{\Omega} \ell_{H}^{c}(x) d \mu(x) ; H \in \mathcal{H}_{N}(\Omega)\right\} .
$$

We start by proving in section 1 the following result.

Theorem 1.1 Let $c$ be a cost function that is continuous and bounded above, then

$$
\operatorname{MK}_{\mathrm{sym}}(c, \mu)=\mathrm{DK}_{\mathrm{sym}}^{1}(c, \mu)=\mathrm{DK}_{\mathrm{sym}}^{2}(c, \mu) .
$$

Moreover, the three extrema are attained.

Of great interest is to determine for which cost functions $c$, problem $\mathrm{MK}_{\mathrm{sym}}(c, \mu)$ is attained at an extremal probability measure $\pi_{0} \in \mathcal{P}_{\text {sym }}\left(\Omega^{N}, \mu\right)$ that is those supported on the graph of the form $x \rightarrow$ $\left(x, S x, S^{2} x, \ldots, S^{N-1} x\right)$, where $S$ is a $\mu$-measure preserving transformation on $\Omega$ such that $S^{N}=I$ a.e. Indeed, it is clear that $\operatorname{MK}_{\mathrm{sym}}(c, \mu) \geq \operatorname{MK}_{\mathrm{cyc}}(c, \mu)$, where

$$
\operatorname{MK}_{\text {сус }}(c, \mu):=\sup \left\{\int_{\Omega^{N}} c\left(x, S x, \ldots, S^{N-1} x\right) d \mu ; S \text { is } \mu \text {-measure preserving on } \Omega \text { and } S^{N}=I \text { a.e. }\right\}
$$

Recently, and after the first version of this paper appeared on arxive, Colombo and Di Marino established the following natural result.

Theorem 1.2 (Colombo-Di Marino) Let $c$ be a cost function that is continuous and bounded above. if $\mu$ has no atoms, then

$$
\operatorname{MK}_{\mathrm{sym}}(c, \mu)=\mathrm{MK}_{\mathrm{cyc}}(c, \mu) \text {. }
$$

In section 2, we give a sufficient condition on the cost function and on the optimal anti-symmetric Hamiltonian $H$ that will insure that $\operatorname{MK}_{\text {cyc }}(c, \mu)$ is attained. 
Theorem 1.3 Let $c$ be a cost function that is continuous and bounded above. Assume that $\operatorname{DK}_{\mathrm{sym}}^{2}(c, \mu)$ is attained at some $H_{\infty} \in \mathcal{H}_{N}(\Omega)$ in such a way that for $\mu$-almost $x \in \Omega$ the map

$$
\left(x_{2}, x_{3}, \ldots, x_{N}\right) \rightarrow c\left(x, x_{2}, \ldots, x_{N}\right)-H_{\infty}\left(x, x_{2}, \ldots, x_{N}\right)
$$

attains its maximum uniquely. Then $\operatorname{MK}_{\text {cyc }}(c, \mu)$ is attained.

Assume now that the cost function itself $c: \Omega^{N} \rightarrow \mathbb{R}$, is itself $\sigma$-symmetric, that is

$$
c\left(x_{1}, x_{2}, \ldots, x_{N}\right)=c\left(x_{2}, x_{3}, \ldots, x_{N}, x_{1}\right) \quad \text { on } \Omega^{N} .
$$

The symmetric Monge-Kantorovich problem is then clearly equivalent to the classical one when all marginals are the same and equal to $\mu$, that is

$$
\operatorname{MK}_{\mathrm{sym}}(c, \mu)=\operatorname{MK}(c ; \mu, \ldots, \mu)=\sup \left\{\int_{\Omega^{N}} c\left(x_{1}, x_{2}, \ldots, x_{N}\right) d \pi ; \pi \in \mathcal{P}\left(\Omega^{N}\right) \& \operatorname{proj}_{i} \pi=\mu, i=1, \ldots, N\right\} .
$$

Examples of such cost functions are:

1. The quadratic cost $c(\mathbf{x})=-\sum_{i=1}^{N} \sum_{j=i+1}^{N}\left|x_{i}-x_{j}\right|^{2}$ considered in full generality by Gangbo-Sweich $[8]$. The symmetric version of the problem, that is when all the marginals are identical, can only admit the trivial solution. In other words the infimum is uniquely attained at the image of $\mu$ by the map $x \rightarrow(x, x, \ldots, x)$, i.e., the involution $S$ is nothing but the identity.

2. The Plakhov cost function $c(x, y)=-1-\cos (x-y)$, which was studied in detail in [15]. This is an example where $\operatorname{MK}_{\mathrm{sym}}(c, \mu)$ does not have a Monge solution, i.e., it is not attained at a measure $\pi_{S}$ that is the image of $\mu$ by a map $x \rightarrow\left(x, S x, S^{2} x, \ldots, S^{N-1} x\right)$, where $S$ is an $N$-involution.

3. The Coulomb $\operatorname{cost} c(\mathbf{x})=-\sum_{i=1}^{N} \sum_{j=i+1}^{N} \frac{1}{\left|x_{i}-x_{j}\right|}$ is a most interesting example since it appears in electronic structure theory. Indeed, recent insight into exchange-correlation in density functional theory led many authors (such as Buttazzo-De Pascale and Gori-Giorgi [2] and Cotar-Friesecke-Klüppelberg [4] to reformulate the electron-electron interaction energy functional with respect to a density $\rho(\mathbf{x})$ as an $N$ dimensional mass transport, where the cost functional is the Newtonian potential

$$
\hat{V}_{e e}=\sum_{i=1}^{N} \sum_{j=i+1}^{N}\left|\mathbf{x}_{\mathbf{i}}-\mathbf{x}_{\mathbf{j}}\right|^{-1},
$$

which correspond to $N$ interacting electrons. Assuming that the admissible configurations of $N$ electrons in $d$-dimensions have the form $\left(\mathbf{f}_{1}(\mathbf{s}), \ldots, \mathbf{f}_{N}(\mathbf{s})\right)$ where $\mathbf{s}$ is a $d$-dimensional vector that determines the position of, say, electron " 1 ", and $\mathbf{f}_{i}(\mathbf{s})\left(i=1, \ldots, N, \mathbf{f}_{1}(\mathbf{s})=\mathbf{s}\right)$ are the co-motion functions, which determine the position of the $i$-th electron in terms of $\mathbf{s}$, and if the variable $\mathbf{s}$ itself is distributed according to the normalized density $\rho(\mathbf{s}) / N$, then the energy functional for "strictly correlated electrons" $V_{e e}^{\mathrm{SCE}}[\rho]$ corresponding to the density $\rho$ is given by the infimum of

$$
\int_{\mathbb{R}^{d}} d \mathbf{s} \frac{\rho(\mathbf{s})}{N} \sum_{i=1}^{N} \sum_{j=i+1}^{N} \frac{1}{\left|\mathbf{f}_{i}(\mathbf{s})-\mathbf{f}_{j}(\mathbf{s})\right|},
$$

among all co-motion functions $\left(\mathbf{f}_{i}\right)_{i}$ that preserve the density $\rho$, so as to ensure the indistinguishability of the $N$ electrons. Formally, such functions must satisfy the equations

$$
\rho\left(\mathbf{f}_{i}(\mathbf{s})\right) d \mathbf{f}_{i}(\mathbf{s})=\rho(\mathbf{s}) d \mathbf{s}, i=1, \ldots, N .
$$

A relaxation of this formulation is to consider $V_{e e}^{\mathrm{SCE}}[\rho]$ as the infimum of

$$
\int_{\mathbb{R}^{N d}} \sum_{i=1}^{N} \sum_{j=i+1}^{N} \frac{1}{\left|x_{i}-x_{j}\right|} d \pi\left(x_{1}, \ldots, x_{N}\right),
$$


over all probability densities on $\mathbb{R}^{N d}$ ("wave functions") whose all marginals are equal to $\frac{\rho}{N}$. The problem posed above, that is searching for the minimum possible interaction energy in a given density, is a typical Monge-Kantorovich problem involving symmetry. The main open problem here is whether there is indeed an optimal co-motion functions $\left(\mathbf{f}_{i}(\mathbf{s})_{i=1}^{N}\right.$ that minimizes both expressions in (12) and (14). If this is the case, then our Lemma 2.3 below shows that the optimal one must be of the form

$$
\mathbf{f}_{1}(\mathbf{s})=\mathbf{s}, \mathbf{f}_{i}(\mathbf{s})=f^{i}(\mathbf{s}) \text { for } i=2, \ldots, N, \text { while } \mathbf{f}^{N+1}(\mathbf{s})=\mathbf{s} \text { for some } f \text { satisfying (13). }
$$

In other words, for each density $\rho$, there exists such an $f$ satisfying (13) such that

$$
V_{e e}^{\mathrm{SCE}}[\rho]=\int_{\mathbb{R}^{d}} d \mathbf{s} \frac{\rho(\mathbf{s})}{N} \sum_{i=1}^{N} \sum_{j=i+1}^{N} \frac{1}{\left|\mathbf{f}^{i}(\mathbf{s})-\mathbf{f}^{j}(\mathbf{s})\right|} .
$$

We note that this has been verified in the case $N=2[2$. The case where there is a higher number of electrons is much more delicate $[5]$.

In this paper, we shall resolve this problem for cost functions on $\Omega^{N}$ of the form

$$
c\left(x_{1}, x_{2}, \ldots, x_{N}\right)=\left\langle u_{1}\left(x_{1}\right), x_{2}\right\rangle+\ldots\left\langle u_{N-1}\left(x_{1}\right), x_{N}\right\rangle
$$

where $u_{1}, \ldots, u_{N-1}$ are given vector fields from $\Omega$ to $\mathbb{R}^{d}$. Note that this cost is not cyclically symmetric, yet we shall optimize it on the class of symmetric probabilities in order to establish an interesting representation for general vector fields in term of cyclically monotone operators. This makes use of a remarkable duality between three fundamental concepts in functional analysis: monotonicity, cyclical symmetry and involutions. Indeed, let $\mathcal{S}(\Omega)$ denote the set of measure preserving transformations on $\Omega$, which can be considered as a closed subset of the sphere of $L^{2}\left(\Omega, \mathbb{R}^{d}\right)$ and let

$$
\mathcal{S}_{N}(\Omega)=\left\{S \in \mathcal{S}(\Omega), S^{N}=I \mu \text { a.e }\right\}
$$

The set $\mathcal{S}_{N}(\Omega)$ has been shown recently [6] to be polar to the class of $N$-cyclically monotone vector fields, which are those $u: \Omega \rightarrow \mathbb{R}^{d}$ that satisfy for every cycle $x_{1}, \ldots, x_{N}, x_{N+1}=x_{1}$ of points in $\Omega$, the inequality

$$
\sum_{i=1}^{N}\left\langle u\left(x_{i}\right), x_{i}-x_{i+1}\right\rangle \geq 0
$$

More generally, Galichon-Ghoussoub [6] introduced the following extension to the case of more than one vector field.

Definition 1.4 A family of vector fields $u_{1}, u_{2}, \ldots, u_{N-1}$ from $\Omega \rightarrow \mathbb{R}^{d}$ is said to be jointly $N-$ monotone if for every cycle $x_{1}, \ldots, x_{2 N-1}$ of points in $\Omega$ such that $x_{N+l}=x_{l}$ for $1 \leq l \leq N-1$, we have

$$
\sum_{i=1}^{N} \sum_{l=1}^{N-1}\left\langle u_{l}\left(x_{i}\right), x_{i}-x_{i+l}\right\rangle \geq 0
$$

Note that if each $u_{\ell}$ is $N$-cyclically monotone, then the family $\left(u_{1}, u_{2}, \ldots, u_{N-1}\right)$ is jointly $N$-monotone. Actually, one needs much less, since the $(N-1)$-tuplet $(u, u, \ldots, u)$ is jointly $N$-monotone if and only if $u$ is 2-monotone. On the other hand, $(u, 0,0, \ldots, 0)$ is jointly $N$-monotone if and only if $u$ is $N$-monotone. See [6] for a complete discussion.

We now state the recent result of Galichon-Ghoussoub [6], which establishes the remarkable duality between $N$-cyclically monotone operators, $N$-antisymmetric Hamiltonians and measure preserving $N$ involutions. We shall also need the notion of an $N$-sub-antisymmetric Hamiltonian on $\Omega$, which is any function $H$ satisfying

$$
\sum_{i=0}^{N-1} H\left(\sigma^{i}\left(x_{1}, \ldots, x_{N}\right)\right) \leq 0 \text { on } \Omega^{N} \text { and } H(x, x, \ldots, x)=0 \text { for all } x \in \Omega .
$$


Theorem 1.5 (Galichon-Ghoussoub) Let $u_{1}, \ldots, u_{N-1}: \Omega \rightarrow \mathbb{R}^{d}$ be bounded measurable vector fields. The following properties are then equivalent:

1. The family $\left(u_{1}, \ldots, u_{N-1}\right)$ is jointly $N$-monotone a.e., that is there exists a measure zero set $\Omega_{0}$ such that $\left(u_{1}, \ldots, u_{N-1}\right)$ is jointly $N$-monotone on $\Omega \backslash \Omega_{0}$.

2. The family $\left(u_{1}, \ldots, u_{N-1}\right)$ is in the polar of $\mathcal{S}_{N}(\Omega, \mu)$ in the following sense,

$$
\inf \left\{\int_{\Omega} \sum_{\ell=1}^{N-1}\left\langle u_{\ell}(x), x-S^{\ell} x\right\rangle d \mu ; S \in \mathcal{S}_{N}(\Omega, \mu)\right\}=0 .
$$

3. There exists a $N$-sub-antisymmetric Hamiltonian $H$ which is concave in the first variable, convex in the last $(N-1)$ variables such that

$$
\left(u_{1}(x), \ldots, u_{N-1}(x)\right)=\nabla_{2, \ldots, N} H(x, x, \ldots, x) \quad \text { for a.e. } x \in \Omega .
$$

Moreover, $H$ is $N$-cyclically antisymmetric in the following sense: For a.e. $\mathbf{x}=\left(x_{1}, \ldots, x_{N}\right) \in \Omega^{N}$, we have

$$
H\left(x_{1}, x_{2}, \ldots, x_{N}\right)+H_{2, \ldots, N}\left(x_{1}, x_{2}, \ldots, x_{N}\right)=0
$$

where $H_{2, \ldots, N}$ is the concavification of the function $K(\mathbf{x})=\sum_{i=1}^{N-1} H\left(\sigma^{i}(\mathbf{x})\right)$ with respect to the last $N-1$ variables.

Note that (19) shows that the above is also equivalent to the statement that

$$
\sup \left\{\int_{\Omega^{N}} \sum_{\ell=1}^{N-1}\left\langle u_{\ell}\left(x_{1}\right), x_{\ell+1}\right\rangle d \pi(\mathbf{x}) ; \pi \in \mathcal{P}_{\mathrm{sym}}\left(\Omega^{N}, \mu\right)\right\}=\int_{\Omega} \sum_{\ell=1}^{N-1}\left\langle u_{\ell}(x), x\right\rangle d \mu(x),
$$

and that the supremum is attained at the image of $\mu$ by the map $x \rightarrow(x, x, \ldots, x)$, which is nothing but a particular case of the symmetric Monge-Kantorovich problem, when the cost function is the one we are considering in (15) and when the family $\left(u_{1}, \ldots, u_{N-1}\right)$ is $N$-monotone.

Theorem 1.6 below can now be seen as the extension of the above, when one considers an arbitrary family of $(N-1)$ vector fields. Indeed, note that in the case of the cost function (15),

$$
\ell_{H}(x)=\sup \left\{\left\langle u_{1}(x), x_{2}\right\rangle+\ldots\left\langle u_{N-1}(x), x_{N}\right\rangle-H\left(x, x_{2}, \ldots, x_{N}\right) ;\left(x_{2}, \ldots, x_{N}\right) \in \Omega^{N-1}\right\},
$$

which means that $\ell_{H}$ is essentially the standard Lagrangian associated to $H$ (i.e., Legendre transform of $H$ with respect to the last $N-1$-variables) and

$$
\ell_{H}(x)=L_{H}\left(x, u_{1}(x), u_{2}(x), \ldots, u_{N-1}(x)\right),
$$

where for $\left(x, p_{1}, \ldots, p_{N-1}\right) \in\left(\mathbb{R}^{d}\right)^{N}$,

$$
L_{H}\left(x, p_{1}, \ldots, p_{N-1}\right)=\sup \left\{\sum_{i=1}^{N-1}\left\langle p_{i}, y_{i}\right\rangle-H\left(x, y_{1}, \ldots, y_{N-1}\right) ; y_{i} \in \Omega\right\} .
$$

The following result will be established in sections 3 and 4 .

Theorem 1.6 Given $(N-1)$ bounded vector fields $u_{1}, u_{2}, \ldots ., u_{N-1}$ from $\Omega$ to $\mathbb{R}^{N}$, and a probability measure $\mu$ on $\Omega$ that is absolutely continuous with respect to Lebesgue measure, we consider the following variational problems:

$$
\begin{aligned}
\operatorname{MK}_{\text {sym }}: & =\sup \left\{\int_{\Omega^{N}}\left[\left\langle u_{1}\left(x_{1}\right), x_{2}\right\rangle+\ldots .\left\langle u_{N-1}\left(x_{1}\right), x_{N}\right\rangle\right] d \pi ; \pi \in \mathcal{P}_{\text {sym }}\left(\Omega^{N}, \mu\right)\right\} . \\
\mathrm{DK}_{\mathrm{sym}}: & =\inf \left\{\int_{\Omega} L_{H}\left(x, u_{1}(x), u_{2}(x), \ldots, u_{N}(x)\right) d \mu(x) ; H \in \mathcal{H}_{N}(\Omega)\right\} . \\
\mathrm{MK}_{\mathrm{cyc}}: & =\sup \left\{\int_{\Omega^{N}}\left[\left\langle u_{1}(x), S x\right\rangle+\left\langle u_{2}(x), S^{2} x\right\rangle+\ldots\left\langle u_{N-1}(x), S^{N-1} x\right\rangle\right] d \mu ; S \in \mathcal{S}_{N}(\Omega)\right\} .
\end{aligned}
$$

If meas $(\partial \Omega)=0$, then the following holds: 
1. $\mathrm{MK}_{\mathrm{sym}}=\mathrm{DK}_{\mathrm{sym}}=\mathrm{MK}_{\mathrm{cyc}}$.

2. $\mathrm{MK}_{\mathrm{cyc}}$ is attained at some $S \in \mathcal{S}_{N}(\Omega)$, which means that $\mathrm{MK}_{\mathrm{sym}}$ is attained at an invariant measure $\pi_{S}$ that is the image of $\mu$ by the map $x \rightarrow\left(x, S x, S^{2} x, \ldots, S^{N-1} x\right)$.

3. There exists a function $H$ on $\mathbb{R}^{d N}$ that is concave in the first variable, convex in the last $(N-1)$ variables and $N$-sub-antisymmetric on $\Omega$, such that

$$
\left(u_{1}(x), \ldots, u_{N-1}(x)\right) \in \partial_{2, \ldots, N} H\left(x, S x, \ldots, S^{N-1} x\right) \quad \text { a.e. } x \in \Omega .
$$

Moreover, if either $u_{i} \in W_{\text {loc }}^{1,1}(\Omega)$ for $i=1,2, \ldots, N-1$ or if $S$ is differentiable a.e., then there exists a $N$-cyclically antisymmetric Hamiltonian $H \in \mathcal{H}_{N}(\Omega)$ such that

$$
\left(u_{1}(x), \ldots, u_{N-1}(x)\right)=\nabla_{2, \ldots, N} H\left(x, S x, \ldots, S^{N-1} x\right) \quad \text { a.e. } x \in \Omega .
$$

4. Assume that for any two families of points $x_{1}, \ldots, x_{N}$ and $y_{1}, \ldots, y_{N}$ in $\Omega$, the function

$$
x \rightarrow \sum_{i=1}^{N-1}\left\langle u_{i}(x), y_{i}-x_{i}\right\rangle+\sum_{i=1}^{N-1}\left\langle u_{i}\left(y_{N-i}\right)-u_{i}\left(x_{N-i}\right), x\right\rangle
$$

has no critical point unless when $x_{1}=y_{1}$. Then there exists a unique measure preserving $N$-involution $S$ such that (26) holds for some concave-convex $N$-sub-antisymmetric Hamiltonian $H$.

If $u: \Omega \rightarrow \mathbb{R}^{d}$ is a single bounded vector field, then the above theorem applied to the family $(0, \ldots, 0, u)$ yields the decomposition

$$
(-u(S x), 0, \ldots, 0, u(x))=\nabla H\left(x, S x, \ldots, S^{N-1} x\right) \quad \text { a.e. } \quad x \in \Omega .
$$

If $S$ is the identity in the above representation, $u$ is then $N$-cyclically monotone, which means that the above theorem essentially says that any bounded vector field is $N$-cyclically monotone up to a measure preserving $N$ - involution. This is clearly in the same spirit as Brenier's theorem stating that any non-degenerate vector field is the gradient of a convex function (i.e., is $N$-cyclically monotone for all $N$ ) modulo a measure preserving transformation. Note that the representation of 2-monotone operators as partial gradients of antisymmetric saddle functions was established by Krause [12. The general version of this result was established in [11] where it is shown that any bounded vector field is 2-monotone up to a measure preserving involution. Theorem 1.6] can be seen as an extension of this result to the case where $N \geq 2$ and where there is more than one vector field.

Actually, in the case of a single vector field $u: \Omega \rightarrow \mathbb{R}^{d}$, one need not consider Hamiltonians on $\Omega^{N}$ as long as the requirement of $N$-antisymmetry is replaced by the following property: Say that a function $F$ on $\mathbb{R}^{d} \times \mathbb{R}^{d}$ is $N$-cyclically sub-antisymmetric on $\Omega$, if

$$
F(x, x)=0 \text { and } \sum_{i=1}^{N} F\left(x_{i}, x_{i+1}\right) \leq 0 \text { for all cyclic families } x_{1}, \ldots, x_{N}, x_{N+1}=x_{1} \text { in } \Omega .
$$

Note that if a function $H\left(x_{1}, \ldots, x_{N}\right)$ is $N$-sub-antisymmetric and if it only depends on the first two variables, then the function $F\left(x_{1}, x_{2}\right):=H\left(x_{1}, x_{2}, \ldots, x_{N}\right)$ is $N$-cyclically sub-antisymmetric.

Our proof then yields the following result.

Theorem 1.7 Consider a vector field $u \in L^{\infty}\left(\Omega, \mathbb{R}^{d}\right)$, then:

1. For every $N \geq 2$, there exists a measure preserving $N$-involution $S$ on $\Omega$ and a globally Lipschitz concave-convex function $F$ of $\mathbb{R}^{d} \times \mathbb{R}^{d}$ that is $N$-cyclically sub-antisymmetric on $\Omega$, such that

$$
(-u(S x), u(x)) \in \partial F(x, S x) \text { for a.e. } x \in \Omega,
$$

where $\partial H$ is the subdifferential of $H$ as a concave-convex function [16]. 
2. If either $u \in W_{l o c}^{1,1}(\Omega)$ or if $S$ is differentiable a.e., then

$$
u(x)=\nabla_{2} F(x, S x) \text { for a.e. } x \in \Omega .
$$

3. Moreover $u$ is $N$-cyclically monotone on $\Omega$ if and only if $S=I$ in the representation (30).

Note that we cannot expect to have a function $F$ such that $\sum_{i=1}^{N} F\left(x_{i}, x_{i+1}\right)=0$ for all cyclic families $x_{1}, \ldots, x_{N}, x_{N+1}=x_{1}$ in $\Omega$. This is the reason why one needs to consider functions of $N$-variables in order to get $N$-antisymmetry as opposed to sub-antisymmetry. Note that the function defined by

$$
H\left(x_{1}, x_{2}, \ldots, x_{N}\right):=\frac{(N-1) F\left(x_{1}, x_{2}\right)-\sum_{i=2}^{N-1} F\left(x_{i}, x_{i+1}\right)}{N},
$$

is $N$-antisymmetric in the sense of belonging to $\mathcal{H}_{N}(\Omega)$ while $H\left(x_{1}, x_{2} \ldots, x_{N}\right) \geq F\left(x_{1}, x_{2}\right)$ on $\Omega^{N}$.

\section{The case of a general cost function}

Let $\mu_{1}, \mu_{2}, \ldots, \mu_{N}$ be a probability measure on a domain $\Omega$ of $\mathbb{R}^{d}$, and consider the following MongeKantorovich problem associated to a given cost function $c: \Omega^{N} \rightarrow \mathbb{R} \cup\{-\infty\}$.

$$
\operatorname{MK}\left(c, \mu_{1}, \ldots, \mu_{N}\right)=\sup \left\{\int_{\Omega^{N}} c\left(x_{1}, x_{2}, \ldots, x_{N}\right) d \pi ; \pi \in \mathcal{P}\left(\Omega^{N}\right) \& \operatorname{proj}_{i} \pi=\mu_{i} \text { for all } i=1, \ldots, N\right\},
$$

where $\mathcal{P}\left(\Omega^{N}\right)$ is the set of probability measures on $\Omega^{N}$. The following proposition is standard.

Proposition 2.1 Assume $c$ is a finitely valued upper semi-continuous and bounded above cost function on $\Omega^{N}$, then

1. There exists $\pi_{0} \in \mathcal{P}\left(\Omega^{N}\right)$ with $\operatorname{proj}_{i} \pi_{0}=\mu_{i}$ for all $i=1, \ldots, N$ where $\operatorname{MK}\left(c, \mu_{1}, \ldots, \mu_{N}\right)$ is attained.

2. The following duality holds: $\operatorname{MK}\left(c, \mu_{1}, \ldots, \mu_{N}\right)$ is equal to

$$
\operatorname{DK}\left(c, \mu_{1}, \ldots, \mu_{N}\right):=\inf \left\{\int_{\Omega^{N}} \sum_{j=1}^{N} u_{j}\left(x_{j}\right) d \mu_{j} ;\left(u_{j}\right)_{j=1}^{N}: \Omega \rightarrow \mathbb{R}^{N} \& \sum_{j=1}^{N} u_{j}\left(x_{j}\right) \geq c\left(x_{1}, \ldots, x_{N}\right)\right\},
$$

and there exists bounded borel functions $u_{1}^{0}, \ldots, u_{N}^{0}$ where $\operatorname{DK}\left(c, \mu_{1}, \ldots, \mu_{N}\right)$ is attained.

We now consider such Monge-Kantorovich problems in the presence of symmetry. Say that $c$ is cyclically symmetric if

$$
c\left(x_{1}, x_{2}, \ldots, x_{N}\right)=c\left(x_{2}, x_{3}, \ldots, x_{N}, x_{1}\right) \text { on } \Omega^{N} .
$$

Proposition 2.2 Assume $c$ is a cyclically symmetric upper semi-continuous and bounded above cost function, and that all marginals $\mu_{i}$ are equal to $\mu$. Then

1. There exists $\tilde{\pi}_{0} \in \mathcal{P}_{\mathrm{sym}}\left(\Omega^{N}\right)$ with $\operatorname{proj}_{i} \pi_{0}=\mu$ for all $i=1, \ldots, N$ where $\operatorname{MK}\left(c, \mu_{1}, \ldots, \mu_{N}\right)$ is attained. Moreover,

$$
\operatorname{MK}\left(\mathrm{c}, \mu_{1}, \ldots, \mu_{\mathrm{N}}\right)=\operatorname{MK}_{\mathrm{sym}}(c, \mu)=\sup \left\{\int_{\Omega^{N}} c\left(x_{1}, x_{2}, \ldots, x_{N}\right) d \pi ; \pi \in \mathcal{P}_{\mathrm{sym}}\left(\Omega^{N}\right) \& \operatorname{proj}_{1} \pi=\mu\right\} .
$$

2. If $c$ is finite, then

$$
\operatorname{MK}_{\mathrm{sym}}(\mathrm{c}, \mu)=\mathrm{DK}_{\mathrm{sym}}^{1}(c, \mu):=\inf \left\{N \int_{\Omega} u(x) d \mu ; u \in C_{b}(\Omega) \text { with } \sum_{j=1}^{N} u\left(x_{j}\right) \geq c\left(x_{1}, \ldots, x_{N}\right)\right\},
$$


and there exists a Borel and bounded function $u_{0}: \Omega \rightarrow \mathbb{R}$ so that

$$
\mathrm{DK}_{\mathrm{sym}}^{1}(c, \mu)=N \int_{\Omega} u_{0}(x) d \mu .
$$

Moreover, $u_{0}$ can be chosen in such a way that

$$
u_{0}(x)=\sup \left\{c\left(x, y_{1}, y_{2}, \ldots, y_{N-1}\right)-\sum_{i=1}^{N-1} u_{0}\left(y_{i}\right)\right\}
$$

Proof: 1. By Proposition 2.1. $\operatorname{MK}(c, \mu, \ldots, \mu)$ is attained at some $\pi_{0} \in \mathcal{P}\left(\Omega^{N}\right)$ with marginals proj ${ }_{i} \pi=\mu$ for $i=1, \ldots, N$. Consider now the probability $\tilde{\pi}_{0}:=\frac{1}{N} \sum_{i=1}^{N} \sigma^{i} \# \pi_{0}$. It is clearly $\sigma$-invariant and with marginal $\mu$. Since $c$ is cyclically symmetric, we have $\int_{\Omega^{N}} c\left(x_{1}, x_{2}, \ldots, x_{N}\right) d \pi_{0}=\int_{\Omega^{N}} c\left(x_{1}, x_{2}, \ldots, x_{N}\right) d \tilde{\pi}_{0}$, meaning that $\tilde{\pi}_{0}$ is also maximizing for both $\operatorname{MK}(c, \mu, \ldots, \mu)$ and $\operatorname{MK}_{\mathrm{sym}}(c, \mu)$.

2. By Proposition 2.1 $\mathrm{DK}(c, \mu)$ is attained at a family of bounded Borel functions $\left(u_{i}^{0}\right)_{i=1}^{N}$. Set

$$
u(x)=\frac{u_{1}^{0}(x)+u_{2}^{0}(x)+\ldots+u_{N}^{0}(x)}{N}
$$

and note that $c$ is cyclically symmetric, $\sum_{j=1}^{N} u\left(x_{j}\right) \geq c\left(x_{1}, \ldots, x_{N}\right)$ and

$$
\mathrm{DK}(c, \mu) \leq \mathrm{DK}_{\mathrm{sym}}^{1}(c, \mu) \leq N \int_{\Omega} u(x) d \mu=\int_{\Omega^{N}} \sum_{j=1}^{N} u\left(x_{j}\right) d \mu=\int_{\Omega^{N}} \sum_{j=1}^{N} u_{j}^{0}\left(x_{j}\right) d \mu=\operatorname{DK}(c ; \mu, \ldots, \mu) .
$$

In order to show (37) we consider the function

$$
\bar{u}(x)=\sup \left\{c\left(x, y_{1}, y_{2}, \ldots, y_{N-1}\right)-\sum_{i=1}^{N-1} u\left(y_{i}\right) ;\left(y_{1}, \ldots, y_{N-1}\right) \in \Omega^{N-1}\right\} .
$$

Since $u$ satisfies the constraint, we have $\bar{u}(x) \leq u(x)$. We now claim that for all $\left(x, x_{2}, \ldots, x_{N}\right)$, we have

$$
\bar{u}(x)+\sum_{i=2}^{N} \bar{u}\left(x_{i}\right) \geq N c\left(x, x_{2}, \ldots, x_{N}\right)-(N-1)\left(\sum_{i=2}^{N} u\left(x_{i}\right)-u(x)\right) .
$$

Indeed, by picking $y_{i}=x_{i+1}$ in the definition of $\bar{u}(x)$, we get that

$$
\bar{u}(x) \geq c\left(x, x_{2}, x_{3}, \ldots, x_{N}\right)-\sum_{i=2}^{N} u\left(x_{i}\right) .
$$

Similary, pick $y_{1}=x, y_{i}=x_{i+1}$ in the definition of $\bar{u}\left(x_{2}\right)$,

$$
\bar{u}\left(x_{2}\right) \geq c\left(x_{2}, x, x_{3}, \ldots, x_{N}\right)-u(x)-\sum_{i=2}^{N} u\left(x_{i}\right)+u\left(x_{2}\right) .
$$

Similary, pick $y_{1}=x, y_{i}=x_{i+1}$ in the definition of $\bar{u}\left(x_{2}\right)$,

$$
\bar{u}\left(x_{3}\right) \geq c\left(x_{3}, x_{2}, x, \ldots, x_{N}\right)-u(x)-\sum_{i=2}^{N} u\left(x_{i}\right)+u\left(x_{3}\right)
$$

Now add up the $\mathrm{N}$ above inequalities and use the fact that $c$ is symmetric to obtain (37). Consider now the function $v(x)=\frac{\bar{u}(x)+(N-1) u(x)}{N}$ in such a way that $\bar{u}(x) \leq v(x) \leq u(x)$. Estimate (37) gives that $\sum_{i=1}^{N} v\left(x_{i}\right) \geq c\left(x_{1}, x_{2}, x_{3}, \ldots, x_{N}\right)$, hence $v$ satisfies the constraint in $\operatorname{DK}_{\mathrm{sym}}^{1}(c, \mu)$. Let now

$$
u_{0}(x)=\inf \left\{w(x) ; \bar{u} \leq w \leq u \& \sum_{i=1}^{N} w\left(x_{i}\right) \geq c\left(x_{1}, x_{2}, x_{3}, \ldots, x_{N}\right)\right\}
$$


The function $u_{0}$ clearly satisfies the constraint in $\operatorname{DK}_{\text {sym }}^{1}(c, \mu)$. Note also that

$$
\begin{aligned}
\bar{u}_{0}(x) & =\sup \left\{c\left(x, y_{1}, y_{2}, \ldots, y_{N-1}\right)-\sum_{i=1}^{N-1} u_{0}\left(y_{i}\right) ;\left(y_{1}, \ldots, y_{N-1}\right) \in \Omega^{N-1}\right\} \\
& \geq \sup \left\{c\left(x, y_{1}, y_{2}, \ldots, y_{N-1}\right)-\sum_{i=1}^{N-1} u\left(y_{i}\right) ;\left(y_{1}, \ldots, y_{N-1}\right) \in \Omega^{N-1}\right\} \\
& =\bar{u}(x),
\end{aligned}
$$

which means that $u \geq \bar{u}_{0} \geq \bar{u}$, If now $\bar{u}_{0}(\bar{x})<u_{0}(\bar{x})$ for some $\bar{x} \in \Omega$, then $u_{0}(\bar{x})>\bar{u}_{0}(\bar{x}) \geq \bar{u}(\bar{x})$, hence contradicting the minimality of $u_{0}$. It follows that $u_{0}=\bar{u}_{0}$, and since $u_{0} \leq u$, it does minimize the functional in $\operatorname{DK}_{\text {sym }}^{1}(c, \mu)$.

We now consider the symmetric Monge-Kantorovich problem when $c$ is not assumed to be cyclically symmetric.

Proof of Theorem 1.1; let $\tilde{c}$ is the symmetrized of $c$ defined for any $\mathbf{x}=\left(x_{1}, x_{2}, \ldots, x_{N}\right)$ by $\tilde{c}(\mathbf{x})=$ $\frac{1}{N} \sum_{i=1}^{N} c\left(\sigma^{i} \mathbf{x}\right)$. It is clear that $\operatorname{MK}_{\mathrm{sym}}(c, \mu)=\operatorname{MK}_{\mathrm{sym}}(\tilde{c}, \mu)$, which by the last proposition is equal to $\mathrm{DK}_{\mathrm{sym}}^{1}(\tilde{c}, \mu)$.

On the other hand, For $H \in \mathcal{H}_{N}(\Omega)$, we let $\ell_{H}^{c}$ be the $c$-Legendre transform of $H$ with respect to the last $(N-1)$ variables, that is

$$
\ell_{H}^{c}(x)=\sup \left\{c\left(x, x_{2}, \ldots, x_{N}\right)-H\left(x, x_{2}, \ldots, x_{N}\right) ;\left(x_{2}, \ldots, x_{N}\right) \in \Omega^{N-1}\right\}
$$

For any invariant probability measure $\pi$, with 1-marginal $\mu$, any $\ell \in L^{1}(\Omega ; \mu)$ and any $H \in \mathcal{H}$, we have

$$
\int_{\Omega^{N}} c\left(x_{1}, x_{2}, \ldots, x_{N}\right) d \pi=\int_{\Omega^{N}}\left[c\left(x_{1}, x_{2}, \ldots, x_{N}\right)-\ell\left(x_{1}\right)-H\left(x_{1}, x_{2}, \ldots, x_{N}\right)\right] d \pi(\mathbf{x})+\int_{\Omega} \ell\left(x_{1}\right) d \mu\left(x_{1}\right),
$$

hence if $\ell\left(x_{1}\right) \geq c\left(x_{1}, \ldots, x_{N}\right)-H\left(x_{1}, \ldots, x_{N}\right)$ for all $\left(x_{1}, x_{2}, \ldots, x_{N}\right)$, then $\int_{\Omega^{N}} c\left(x_{1}, x_{2}, \ldots, x_{N}\right) d \pi \leq \int_{\Omega} \ell(x) d \mu(x)$, and therefore $\mathrm{MK}_{\mathrm{sym}}(c, \mu) \leq \mathrm{DK}_{\mathrm{sym}}^{2}(c, \mu)$.

For the reverse inequality, we shall use the fact that

$$
\operatorname{MK}_{\mathrm{sym}}(c, \mu)=\operatorname{MK}_{\mathrm{sym}}(\tilde{c}, \mu)=\operatorname{DK}_{\mathrm{sym}}^{1}(\tilde{c}, \mu)=N \int_{\Omega} u_{0}(x) d \mu,
$$

where $u_{0}$ is a lower semi-continuous function satisfying

$$
u_{0}(x)=\sup \left\{\tilde{c}\left(x, y_{1} \ldots, y_{N-1}\right)-\sum_{i=1}^{N-1} u_{0}\left(y_{i}\right): y_{1}, \ldots, y_{N-1} \in \mathbb{R}^{d}\right\} .
$$

Notice that $N u_{0}=\ell_{H}^{\tilde{c}}$, where $H$ is the $N$-cyclically symmetric Hamiltonian defined by

$$
H\left(x_{1}, x_{2}, \ldots, x_{N}\right):=\sum_{i=2}^{N} u_{0}\left(x_{i}\right)-(N-1) u_{0}\left(x_{1}\right) .
$$

Finally, it is easy to check that $N u_{0}=\ell_{H_{\infty}}^{c}$, where $H_{\infty}$ is the $N$-cyclically symmetric Hamiltonian

$$
H_{\infty}\left(x_{1}, x_{2}, \ldots, x_{N}\right):=-\frac{1}{N} \sum_{i=1}^{N} c\left(\sigma^{i}\left(x_{1}, x_{2}, \ldots, x_{N}\right)\right)+c\left(x_{1}, x_{2}, \ldots, x_{N}\right)+\sum_{i=2}^{N} u_{0}\left(x_{i}\right)-(N-1) u_{0}\left(x_{1}\right) .
$$

It follows that

$$
\operatorname{MK}_{\mathrm{sym}}(c, \mu)=N \int_{\Omega} u_{0}(x) d \mu=\mathrm{DK}_{\mathrm{sym}}^{2}(c, \mu)
$$

and that the latter is attained at $H_{\infty}$. This completes the proof of Theorem 1.1

Proof of Theorem 1.3; This will follow from Theorem 1.1 combined with the following three lemmas. 
Lemma 2.1 Let $L:=\ell_{H_{\infty}}^{c}$ where $H_{\infty}$ is a fixed Hamiltonian in $\mathcal{H}_{N}(\Omega)$, and let $x \in \Omega$ be such that $\left(x_{2}, x_{3}, \ldots, x_{N}\right) \rightarrow c\left(x, x_{2}, \ldots, x_{N}\right)-H_{\infty}\left(x, x_{2}, \ldots, x_{N}\right)$ attains its maximum uniquely at $S_{1} x, S_{2} x, \ldots, S_{N-1} x$. Let $H \in \mathcal{H}, r \in \mathbb{R}$ and consider $L_{r}^{c}:=\ell_{H_{\infty}+r H}^{c}$ to be the c-Legendre transform associated to the Hamiltonian $H_{\infty}+r H$. Then, we have

$$
\lim _{r \rightarrow 0} \frac{L_{r}(x)-L(x)}{r}=H\left(x, S_{1} x, S_{2} x, \ldots, S_{N-1} x\right) .
$$

Proof: Let $\left(x_{1, r}, \ldots, x_{N-1, r}\right)$ be points in $\Omega$, where

$$
\left(x_{2}, x_{3}, \ldots, x_{N}\right) \rightarrow c\left(x, x_{2}, \ldots, x_{N}\right)-H_{\infty}\left(x, x_{2}, \ldots, x_{N}\right)-r H\left(x, x_{2}, \ldots, x_{N}\right)
$$

attains its maximum. It follows that

$$
\begin{aligned}
L_{r}(x)-L(x)= & c\left(x, x_{1, r}, \ldots, x_{N-1, r}\right)-H_{\infty}\left(x, x_{1, r}, \ldots, x_{N-1, r}\right)-r H\left(x, x_{1, r}, \ldots ., x_{N-1, r}\right) \\
& -c\left(x, S_{1} x, S_{2} x, \ldots, S_{N-1} x\right)+H_{\infty}\left(x, S_{1} x, S_{2} x, \ldots, S_{N-1} x\right),
\end{aligned}
$$

and therefore

$$
-H_{\infty}\left(x, S_{1} x, S_{2} x, \ldots, S_{N-1} x\right) \leq \frac{L_{r}(x)-L(x)}{r} \leq-H\left(x, x_{1, r}, \ldots, x_{N-1, r}\right) .
$$

Since $S_{1} x, \ldots, S_{2} x$ are unique maxima, it follows that as $r \rightarrow 0$, we have that $x_{i, r}$ converges to $S_{i} x$, from which we conclude that $\lim _{r \rightarrow 0} \frac{L_{r}(x)-L(x)}{r}=H\left(x, S_{1} x, S_{2} x, \ldots, S_{N-1} x\right)$.

Lemma 2.2 Assume that

$$
\operatorname{DK}_{\mathrm{sym}}^{2}(c, \mu):=\inf \left\{\int_{\Omega} \ell_{H}^{c}(x) d \mu(x) ; H \in \mathcal{H}_{N}(\Omega)\right\}
$$

is attained at some $H_{\infty} \in \mathcal{H}_{N}(\Omega)$, and that for $\mu$-almost $x \in \Omega$ the map

$$
\left(x_{2}, x_{3}, \ldots, x_{N}\right) \rightarrow c\left(x, x_{2}, \ldots, x_{N}\right)-H_{\infty}\left(x, x_{2}, \ldots, x_{N}\right)
$$

attains its maximum uniquely at $S_{1} x, S_{2} x, \ldots, S_{N-1} x$. Then, for any $H \in \mathcal{H}_{N}(\Omega)$, we have

$$
\int_{\Omega} H\left(x, S_{1} x, S_{2} x, \ldots, S_{N-1} x\right) d \mu=0 .
$$

Proof: Let $L=\ell_{H_{\infty}}^{c}$. For any $N$-symmetric Hamiltonian $H$ and $r \in \mathbb{R}$, consider $L_{r}^{c}=\ell_{H_{\infty}+r H}^{c}$ to be the $c$-Legendre transform associated to the Hamiltonian $H_{\infty}+r H$. The above lemma yields that for $\mu$-almost all $x \in \Omega$, we have

$$
\lim _{r \rightarrow 0} \frac{L_{r}(x)-L(x)}{r}=H\left(x, S_{1} x, S_{2} x, \ldots, S_{N-1} x\right) .
$$

On the other hand, the extremality of $L:=\ell_{H_{\infty}}$ gives that

$$
0=\lim _{r \rightarrow 0} \int_{\Omega} \frac{L_{r}(x)-L(x)}{r} d \mu=\int_{\Omega} H\left(x, S_{1} x, S_{2} x, \ldots, S_{N-1} x\right) .
$$

At the core of our results is the following duality between $N$-antisymmetric Hamiltonians and measure preserving $N$-involutions, which will be crucial to what follows.

Lemma 2.3 Let $S_{1}, S_{2}, \ldots, S_{N-1}$ be $\mu$-measurable maps on $\Omega$. The following statements are then equivalent:

1. $\int_{\Omega} H\left(x, S_{1} x, S_{2} x, \ldots, S_{N-1} x\right) d \mu=0$ for any $N$-cyclically symmetric Hamiltonian $H$.

2. There exists $S: \Omega \rightarrow \Omega$, $\mu$-measure preserving such that $S^{N}=I$ and $S^{i}=S_{i}$ for all $i=1, \ldots, N-1$. 
Proof: If $S$ is $\mu$-measure preserving and $S^{N}=I$ a.e., then

$$
\int_{\Omega} H\left(x, S x, S^{2} x, \ldots S^{N-1} x\right) d \mu=\int_{\Omega} H\left(S x, S^{2} x, \ldots S^{N-1} x, x\right) d \mu=\ldots=\int_{\Omega} H\left(S^{N-1} x, \ldots, S^{2} x, x, S x\right) d \mu
$$

Since $H$ is $N$-symmetric, then

$$
H\left(x, S x, S^{2} x, \ldots S^{N-1} x\right)+H\left(S x, S^{2} x, \ldots S^{N-1} x, x\right)+\ldots H\left(S^{N-1} x, \ldots, S^{2} x, x, S x\right)=0 .
$$

It follows that $\int_{\Omega} H\left(x, S x, S^{2} x, \ldots S^{N-1} x\right) d \mu=0$.

For the reverse implication, assume $\int_{\Omega} H\left(x, S_{1} x, S_{2} x, \ldots, S_{N-1} x\right) d \mu=0$ for any $N$-cyclically symmetric Hamiltonian $H$. By using the identity with Hamiltonians $\left(H_{i}\right)_{i=1}^{N}$ of the form

$$
H_{i}\left(x_{1}, x_{2}, \ldots, x_{N}\right)=f\left(x_{1}\right)-f\left(x_{i}\right),
$$

where $f$ is a continuous function, one gets that $S_{i}$ is measure preserving for each $i=1, \ldots, N-1$.

Now take for each fixed $i=1, \ldots, N$, the Hamiltonian

$$
H_{i}\left(x_{1}, x_{2}, \ldots, x_{N}\right)=\left|x_{i}-S_{1}^{i} x_{N}\right|-\left|S_{1}^{i} x_{1}-x_{i+1}\right|-\left|x_{i+1}-S_{1}^{i} x_{1}\right|+\left|S_{1}^{i} x_{2}-x_{i+2}\right| .
$$

We have that $H_{i} \in \mathcal{H}_{N}(\Omega)$ for each $i$, since it is of the form $H_{i}\left(x_{1}, \ldots, x_{N}\right)=f\left(x_{1}, x_{i}, x_{N}\right)-f\left(x_{2}, x_{i+1}, x_{1}\right)$. Hence, for each $i$,

$$
0=\int_{\Omega} H_{i}\left(x, S_{1} x, S_{2} x, \ldots, S_{N-1} x\right) d \mu=0=\int_{\Omega}\left(\left(\left|S_{i-1} x-S_{1}^{i} S_{N-1}\right|+\left|S_{1}^{i} S_{1} x-S_{i+1} x\right|\right) d \mu=0 .\right.
$$

It follows that $S_{i+1}=S_{1}^{i+1}$ and $S_{i-1} x=S_{1}^{i} S_{N-1}$ for each $i=1, \ldots, N$. The latter applied to $i=1$, yields $x=S_{1} S_{N-1}=S_{1} S_{1}^{N-1}=S_{1}^{N}$, and we are done.

\section{Concave-convexification of $N$-antisymmetric functions}

Let $\Omega$ be a bounded domain in $\mathbb{R}^{d}$, and consider the class

$$
\mathcal{H}_{N}^{-}(\Omega):=\left\{H \in C\left(\bar{\Omega}^{N}\right) ; \sum_{i=0}^{N-1} H\left(\sigma^{i}(\mathbf{x})\right) \leq 0 \text { for all } \mathbf{x} \in \Omega^{N}\right\} .
$$

For each $H \in \mathcal{H}_{N}^{-}(\Omega)$, we associate the following functional on $\Omega \times\left(\mathbb{R}^{d}\right)^{N-1}$,

$$
L_{H}\left(x, p_{1}, \ldots, p_{N-1}\right)=\sup \left\{\sum_{i=1}^{N-1}\left\langle p_{i}, y_{i}\right\rangle-H\left(x, y_{1}, \ldots, y_{N-1}\right) ; y_{i} \in \Omega\right\} .
$$

Denote by

$$
\mathcal{L}_{-}(N)=\left\{L_{H} ; H \in \mathcal{H}_{N}^{-}(\Omega)\right\} .
$$

Our plan is to show that one can associate to $H$,

- a globally Lipschitz-continuous function $H_{\text {reg }}^{1} \in \mathcal{L}_{-}(N)$ that is concave in the first variable, convex in the last $(N-1)$ variables such that $L_{H_{r e g}^{1}} \leq L_{H}$.

- a globally Lipschitz-continuous function $H_{\text {reg }}^{2} \in \mathcal{L}(N)$ such that $H_{r e g}^{2} \geq H_{\text {reg }}^{1}$ and hence

$$
L_{H_{r e g}^{2}} \leq L_{H_{r e g}^{1}} \leq L_{H}
$$


Suppose that $\Omega$ is contained in a ball $B_{R}$ centered at the origin with radius $R>0$ in $\mathbb{R}^{d}$, we define "an $\left(\bar{\Omega} \times B_{R}\right)$ restricted Legendre transform" of $L_{H}$ as

$$
L_{H}^{*}\left(p_{1}, \ldots, p_{N-1}, x\right)=\sup _{q \in \bar{\Omega}, y_{i} \in B_{R}}\left\{\langle q, x\rangle+\sum_{i=1}^{N-1}\left\langle p_{i}, y_{i}\right\rangle-L_{H}\left(q, y_{1}, y_{2}, \ldots, y_{N-1}\right)\right\} .
$$

Similarly, we define on $\mathbb{R}^{d} \times\left(\mathbb{R}^{d}\right)^{N-1}$,

$$
L_{H}^{* *}\left(x, p_{1}, \ldots, p_{N-1}\right)=\sup _{p \in \bar{\Omega}, x_{i} \in B_{R}}\left\{\langle x, p\rangle+\sum_{i=1}^{N-1}\left\langle p_{i}, x_{i}\right\rangle-L_{H}^{*}\left(x_{1}, \ldots, x_{N-1}, p\right)\right\} .
$$

For any function $L: \mathbb{R}^{d} \times\left(\mathbb{R}^{d}\right)^{N-1} \rightarrow \mathbb{R}$, we define its " $B_{R}$-Hamiltonian" by

$$
H_{L}\left(x, y_{1}, \ldots, y_{N-1}\right)=\sup _{p_{i} \in B_{R}}\left\{\sum_{i=1}^{N-1}\left\langle p_{i}, y_{i}\right\rangle-L\left(x, p_{1}, \ldots, p_{N-1}\right)\right\} .
$$

Finally, for $H \in \mathcal{H}_{N}^{-}(\Omega)$, we consider the following two regularizations of $H$ :

$$
H_{r e g}^{1}(\mathbf{x})=H_{L_{H}^{* *}}(\mathbf{x}),
$$

and

$$
H_{r e g}^{2}(\mathbf{x})=\frac{(N-1) H_{r e g}^{1}(\mathbf{x})-\sum_{i=1}^{N-1} H_{r e g}^{1}\left(\sigma^{i}(\mathbf{x})\right)}{N} .
$$

We list some of the properties of $H_{r e g}^{1}, H_{r e g}^{2}, L_{H_{r e g}}^{1}$ and $L_{H_{r e g}}^{1}$.

Proposition 3.1 If $H \in \mathcal{H}_{N}^{-}(\Omega)$, then the following statements hold:

1. $H_{\text {reg }}^{1}$ is a concave-convex on $\mathbb{R}^{d} \times \mathbb{R}^{d(N-1)}$ whose restriction to $\bar{\Omega}^{N}$ belong to $\mathcal{H}_{N}^{-}(\Omega)$.

2. $H_{\text {reg }}^{2}$ belongs to $\mathcal{H}_{N}(\Omega)$, and $H_{\text {reg }}^{2} \geq H_{\text {reg }}^{1}$ on $\bar{\Omega}^{N}$.

3. $L_{H_{r e g}^{1}}$ is convex and continuous in all variables and $L_{H_{r e g}^{2}} \leq L_{H_{r e g}^{1}} \leq L_{H}$ on $\bar{\Omega} \times\left(B_{R}\right)^{N-1}$.

4. $\left|L_{H_{r e g}^{1}}\left(x, p_{1}, \ldots, p_{N-1}\right)\right| \leq R\|x\|+R \sum_{i=1}^{N-1}\left\|p_{i}\right\|+(2 N+1) R^{2}$ for all $x$ and all $\left(p_{i}\right)_{i=1}^{N-1}$ in $\mathbb{R}^{d}$.

5. $\left|H_{r e g}^{1}\left(x, y_{1}, \ldots, y_{N-1}\right)\right| \leq R\|x\|+R \sum_{i=1}^{N-1}\left\|y_{i}\right\|+2 N R^{2}$ for all $x$ and all $\left(y_{i}\right)_{i=1}^{N-1}$ in $\mathbb{R}^{d}$.

6. $L_{H_{r e g}^{2}}$ and $H_{r e g}^{2}$ are both Lipschitz continuous with Lipschitz constants less than $4 N R$.

The proof will require several lemmas.

Lemma 3.1 With the above notation, we have the following properties:

1. $L_{H}^{* *}\left(x, p_{1}, \ldots, p_{N-1}\right) \leq L_{H}\left(x, p_{1}, \ldots, p_{N-1}\right)$ for $x \in \bar{\Omega}$ and $p_{i} \in \mathbb{R}^{d}$ for $i=1, \ldots, N-1$.

2. If $H_{\mathrm{reg}}^{1}$ denotes $H_{L_{H}^{* *}}$, then $H_{\mathrm{reg}}^{1}$ is concave in the first variable and convex in the last $(N-1)$ variables.

3. $L_{H_{\mathrm{reg}}^{1}}$ is jointly convex in all variables. 
Proof. 1) For $x \in \bar{\Omega}$ and $p_{i} \in \mathbb{R}^{d}, i=1, \ldots, N-1$, we have we have

$$
\begin{aligned}
L_{H}^{* *}\left(x, p_{1}, \ldots, p_{N-1}\right) & =\sup _{q \in \bar{\Omega}, r_{i} \in B_{R}}\left\{\langle x, q\rangle+\sum_{i=1}^{N-1}\left\langle p_{i}, r_{i}\right\rangle-L_{H}^{*}\left(r_{1}, \ldots, r_{N-1}, q\right)\right\} \\
& =\sup _{q \in \bar{\Omega}, r_{i} \in B_{R}}\left\{\langle x, q\rangle+\sum_{i=1}^{N-1}\left\langle p_{i}, r_{i}\right\rangle-\sup _{y \in \bar{\Omega}, y_{i} \in B_{R}}\left\{\langle y, q\rangle+\sum_{i=1}^{N-1}\left\langle r_{i}, y_{i}\right\rangle-L_{H}\left(y, y_{1}, \ldots, y_{N-1}\right)\right\}\right\} \\
& =\sup _{q \in \bar{\Omega}, r_{i} \in B_{R}} \inf _{y \in \bar{\Omega}, y_{i} \in B_{R}}\left\{\langle x, q\rangle+\sum_{i=1}^{N-1}\left\langle p_{i}, r_{i}\right\rangle-\langle y, q\rangle-\sum_{i=1}^{N-1}\left\langle r_{i}, y_{i}\right\rangle+L_{H}\left(y, y_{1}, \ldots, y_{N-1}\right)\right\} \\
& =\sup _{q \in \bar{\Omega}, r_{i} \in B_{R}} y \in \bar{\Omega}, y_{i} \in B_{R} \\
& =\sup _{q \in \bar{\Omega}, r_{i} \in B_{R}}\left\{\left\langle q \in \bar{\Omega}, y_{i} \in B_{R}\{y\rangle+\sum_{i=1}^{N-1}\left\langle p_{i}-y_{i}, r_{i}\right\rangle+L_{H}\left(y, y_{1}, \ldots, y_{N-1}\right)\right\}\right. \\
& =\sup _{q \in \bar{\Omega}, r_{i} \in B_{R}}\left\{\inf _{y \in \bar{\Omega}, y_{i} \in B_{R}} \sup _{t_{i} \in \Omega}\left\{\langle q, x-y\rangle+\sum_{i=1}^{N-1}\left\langle p_{i}-y_{i}, r_{i}\right\rangle+\sum_{i=1}^{N-1}\left\langle t_{i}, y_{i}\right\rangle-H\left(y, t_{1}, \ldots, t_{N-1}\right)\right\}\right. \\
& =\inf _{y \in \bar{\Omega}, y_{i} \in B_{R}} \sup _{q \in \bar{\Omega}, r_{i} \in B_{R}} \sup _{t_{i} \in \Omega}\left\{\langle q, x-y\rangle+\sum_{i=1}^{N-1}\left\langle p_{i}-y_{i}, r_{i}\right\rangle+\sum_{i=1}^{N-1}\left\langle t_{i}, y_{i}\right\rangle-H\left(y, t_{1}, \ldots, t_{N-1}\right)\right\} .
\end{aligned}
$$

By taking $y=x$ and $y_{i}=p_{i}$, we readily get that $L_{H}^{* *}\left(x, p_{1}, \ldots, p_{N-1}\right) \leq L_{H}\left(x, p_{1}, \ldots, p_{N-1}\right)$.

For 2) note first that by definition

$$
H_{L^{* *}}\left(x, y_{1}, \ldots, y_{N-1}\right)=\sup _{p_{i} \in B_{R}}\left\{\sum_{i=1}^{N-1}\left\langle p_{i}, y_{i}\right\rangle-L_{H}^{* *}\left(x, p_{1}, \ldots, p_{N-1}\right)\right\},
$$

and therefore for all $x \in \mathbb{R}^{d}$, the function $\left(y_{1}, \ldots, y_{N-1}\right) \rightarrow H_{L^{* *}}\left(x, y_{1}, \ldots, y_{N-1}\right)$ is convex. We shall show that for all $\left(y_{1}, \ldots, y_{N-1}\right) \in\left(\mathbb{R}^{d}\right)^{N-1}$, the function $x \rightarrow H_{L^{* *}}\left(x, y_{1}, \ldots, y_{N-1}\right)$ is concave. In fact we show that

$$
x \rightarrow-H_{L^{* *}}\left(x, y_{1}, \ldots, y_{N-1}\right)=\inf _{p_{i} \in B_{R}}\left\{L_{H}^{* *}\left(x, p_{1}, \ldots, p_{N-1}\right)-\sum_{i=1}^{N-1}\left\langle p_{i}, y_{i}\right\rangle\right\}
$$

is convex. Indeed, consider $\lambda \in(0,1)$ and elements $x_{1}, x_{2} \in \mathbb{R}^{d}$, then for any $a, b$ such that

$$
a>-H_{L^{* *}}\left(x_{1}, y_{1}, \ldots, y_{N-1}\right) \text { and } b>-H_{L^{* *}}\left(x_{2}, y_{1}, \ldots, y_{N-1}\right),
$$

we can find $\left(r_{i}\right)_{i=1}^{N-1}$ and $\left(q_{i}\right)_{i=1}^{N-1}$ in $\left(\mathbb{R}^{d}\right)^{N-1}$ such that

$$
-H_{L^{* *}}\left(x_{1}, y_{1}, \ldots, y_{N-1}\right) \leq L_{H}^{* *}\left(x_{1}, r_{1}, \ldots, r_{N-1}\right)-\sum_{i=1}^{N-1}\left\langle r_{i}, y_{i}\right\rangle \leq a,
$$

and

$$
-H_{L^{* *}}\left(x_{2}, y_{1}, \ldots, y_{N-1}\right) \leq L_{H}^{* *}\left(x_{2}, q_{1}, \ldots, q_{N-1}\right)-\sum_{i=1}^{N-1}\left\langle q_{i}, y_{i}\right\rangle \leq b .
$$


Use the convexity of the ball $B_{R}$ and the convexity of the function $L_{H}^{* *}$ in both variables to write

$$
\begin{aligned}
-H_{L_{H}^{* *}}\left(\lambda x_{1}+(1-\lambda) x_{2}, y_{1}, \ldots, y_{N-1}\right)= & \inf _{p_{i} \in B_{R}}\left\{L_{H}^{* *}\left(\lambda x_{1}+(1-\lambda) x_{2}, p_{1}, \ldots, p_{N-1}\right)-\sum_{i=1}^{N-1}\left\langle p_{i}, y_{i}\right\rangle\right\} \\
\leq & \left.L_{H}^{* *}\left(\lambda x_{1}+(1-\lambda) x_{2}, \lambda r_{1}+(1-\lambda) q_{1}, \ldots, \lambda r_{N-1}+(1-\lambda) q_{N-1}\right)\right) \\
& -\sum_{i=1}^{N-1}\left\langle\lambda r_{i}+(1-\lambda) q_{i}, y_{i}\right\rangle \\
\leq & \lambda\left(L_{H}^{* *}\left(x_{1}, r_{1}, \ldots, r_{N-1}\right)-\sum_{i=1}^{N-1}\left\langle r_{i}, y_{i}\right\rangle\right) \\
& \left.+(1-\lambda)\left(L_{H}^{* *}\left(x_{2}, q_{1}, \ldots, q_{N-1}\right)-\sum_{i=1}^{N-1}\left\langle q_{i}, y_{i}\right\rangle\right)\right\} \\
\leq & \lambda a+(1-\lambda) b,
\end{aligned}
$$

which establishes the concavity of $x \rightarrow H_{L_{H}^{* *}}\left(x, y_{1}, \ldots, y_{N-1}\right)$. It then follows that $L_{H_{r e g}^{1}}=L_{H_{L_{H}^{* *}}}$ is convex in all variables that proves part 3 ).

Lemma 3.2 If $H \in \mathcal{H}_{N}^{-}(\Omega)$, then $H_{\mathrm{reg}}^{1} \in \mathcal{H}_{N}^{-}(\Omega)$.

Proof. Let $i, j=1,2, . ., N$. We first show that

$$
\sum_{i=1}^{N}\left\{\sum_{j=1, j \neq i}^{N}\left\langle p_{j}^{i}, x_{j}\right\rangle-L_{H}^{* *}\left(R^{i-1}\left(p_{1}^{i}, \ldots, p_{i-1}^{i}, x_{i}, p_{i+1}^{i}, \ldots, p_{N}^{i}\right)\right)\right\} \leq 0,
$$

for all $x_{i} \in \Omega$ and $p_{j}^{i} \in \mathbb{R}^{d}$. Indeed, we have

$$
\begin{aligned}
L_{H}\left(\sigma^{i-1}\left(p_{1}^{i}, \ldots, p_{i-1}^{i}, x_{i}, p_{i+1}^{i}, \ldots, p_{N}^{i}\right)\right) & =\sup \left\{\sum_{j=1, j \neq i}^{N}\left\langle p_{j}^{i}, y_{j}\right\rangle-H\left(\sigma^{i-1}\left(y_{1}, \ldots, y_{i-1}, x_{i}, y_{i+1}, \ldots, y_{N}\right)\right) ; y_{j} \in \Omega\right\} \\
& \geq \sum_{j=1, j \neq i}^{N}\left\langle p_{j}^{i}, x_{j}\right\rangle-H\left(\sigma^{i-1}\left(x_{1}, x_{2}, \ldots, x_{n}\right)\right) .
\end{aligned}
$$

Taking summation over $i$ implies that

$$
\sum_{i=1}^{N} L_{H}\left(\sigma^{i-1}\left(p_{1}^{i}, \ldots, p_{i-1}^{i}, x_{i}, p_{i+1}^{i}, \ldots, p_{N}^{i}\right)\right) \geq \sum_{i=1}^{N} \sum_{j=1, j \neq i}^{N}\left\langle p_{j}^{i}, x_{j}\right\rangle-\sum_{i=1}^{N} H\left(\sigma^{i-1}\left(x_{1}, x_{2}, \ldots, x_{n}\right)\right)
$$

Since $\sum_{i=1}^{N} H\left(\sigma^{i-1}\left(x_{1}, x_{2}, \ldots, x_{n}\right)\right) \leq 0$, we obtain

$$
\sum_{i=1}^{N} L_{H}\left(\sigma^{i-1}\left(p_{1}^{i}, \ldots, p_{i-1}^{i}, x_{i}, p_{i+1}^{i}, \ldots, p_{N}^{i}\right)\right) \geq \sum_{i=1}^{N} \sum_{j=1, j \neq i}^{N}\left\langle p_{j}^{i}, x_{j}\right\rangle .
$$

It follows from the definition of $L_{H}^{* *}$ that

$$
\sum_{i=1}^{N} L_{H}^{* *}\left(\sigma^{i-1}\left(p_{1}^{i}, \ldots, p_{i-1}^{i}, x_{i}, p_{i+1}^{i}, \ldots, p_{N}^{i}\right)\right) \geq \sum_{i=1}^{N} \sum_{j=1, j \neq i}^{N}\left\langle p_{j}^{i}, x_{j}\right\rangle .
$$

By moving the left hand side expression to the the other side, we have

$$
0 \geq \sum_{i=1}^{N}\left\{\sum_{j=1, j \neq i}^{N}\left\langle p_{j}^{i}, x_{j}\right\rangle-L_{H}^{* *}\left(\sigma^{i-1}\left(p_{1}^{i}, \ldots, p_{i-1}^{i}, x_{i}, p_{i+1}^{i}, \ldots, p_{N}^{i}\right)\right)\right\} .
$$


Taking sup over all $p_{i}^{j} \in B_{R}$ we obtain $\sum_{i=1}^{N} H_{L_{H}^{* *}}\left(\sigma^{i-1}\left(x_{1}, x_{2}, \ldots, x_{n}\right)\right) \leq 0$ and we are done.

We now recall the following standard elementary result.

Lemma 3.3 Let $D$ be an open set in $\mathbb{R}^{m}$ such that $\bar{D} \subset \tilde{B}_{R}$ where $\tilde{B}_{R}$ is ball with radious $R$ centered at the origin in $\mathbb{R}^{m}$. Let $f: \mathbb{R}^{m} \rightarrow \mathbb{R}$ and define $\tilde{f}: \mathbb{R}^{m} \rightarrow \mathbb{R}$ by

$$
\tilde{f}(y)=\sup _{z \in D}\{\langle y, z\rangle-f(z)\} .
$$

If $f \in L^{\infty}(D)$, then $\tilde{f}$ is a convex Lipschitz function and

$$
\left|\tilde{f}\left(y_{1}\right)-\tilde{f}\left(y_{2}\right)\right| \leq R\left\|y_{1}-y_{2}\right\| \text { for all } y_{1}, y_{2} \in \mathbb{R}^{m} .
$$

Lemma 3.4 If $H \in \mathcal{H}_{N}^{-}(\Omega)$, then the following statements hold:

1. $\left|L_{H}^{* *}\left(x, p_{1}, \ldots, p_{N-1}\right)\right| \leq R\|x\|+R \sum_{i=1}^{N-1}\left\|p_{i}\right\|+(2 N-1) R^{2}$ for all $x$ and $\left(p_{i}\right)_{i=1}^{N-1}$ in $\mathbb{R}^{d}$.

2. $\left|H_{L_{H}^{* *}}\left(x, y_{1}, \ldots, y_{N-1}\right)\right| \leq R\|x\|+R \sum_{i=1}^{N-1}\left\|y_{i}\right\|+2 N R^{2}$ for all $x$ and $\left(y_{i}\right)_{i=1}^{N-1}$ in $\mathbb{R}^{d}$.

3. $L_{H}^{* *}$ and $H_{L_{H}^{* *}}$ are Lipschitz continuous with Lipschitz constants $\operatorname{Lip}\left(H_{L_{H}^{* *}}\right), \operatorname{Lip}\left(L_{H}^{* *}\right) \leq N R$.

Proof. Since $H$ is $N$-sub-antisymmetric, we have $H(x, \ldots, x) \leq 0$, hence

$$
L_{H}\left(x, p_{1}, \ldots, p_{N-1}\right) \geq \sum_{i=1}^{N-1}\left\langle p_{i}, x\right\rangle \quad \text { on } \bar{\Omega} \times\left(\mathbb{R}^{d}\right)^{N-1} .
$$

This together with the fact that $\bar{\Omega} \subset B_{R}$ imply that

$$
\begin{aligned}
L_{H}^{*}\left(p_{1}, \ldots, p_{N-1}, x\right) & =\sup _{q \in \bar{\Omega}, y_{i} \in B_{R}}\left\{\langle q, x\rangle+\sum_{i=1}^{N-1}\left\langle p_{i}, y_{i}\right\rangle-L_{H}\left(q, y_{1}, y_{2}, \ldots, y_{N-1}\right)\right\} . \\
& \leq \sup _{q \in \bar{\Omega}, y_{i} \in B_{R}}\left\{\langle q, x\rangle+\sum_{i=1}^{N-1}\left\langle p_{i}, y_{i}\right\rangle-\sum_{i=1}^{N-1}\left\langle q, y_{i}\right\rangle\right\} . \\
& \leq R\|x\|+R \sum_{i=1}^{N-1}\left\|p_{i}\right\|+(N-1) R^{2} .
\end{aligned}
$$

With a similar argument we obtain that $L_{H}^{* *}\left(x, p_{1}, \ldots, p_{N-1}\right) \leq R\|x\|+R \sum_{i=1}^{N-1}\left\|p_{i}\right\|+(N-1) R^{2}$. We also have

$$
\begin{aligned}
L_{H}^{* *}\left(x, p_{1}, \ldots, p_{N-1}\right) & =\sup _{p \in \bar{\Omega}, x_{i} \in B_{R}}\left\{\langle x, p\rangle+\sum_{i=1}^{N-1}\left\langle p_{i}, x_{i}\right\rangle-L_{H}^{*}\left(x_{1}, \ldots, x_{N-1}, p\right)\right\} \\
& \geq\langle x, p\rangle+\sum_{i=1}^{N-1}\left\langle p_{i}, x_{i}\right\rangle-L_{H}^{*}\left(x_{1}, \ldots, x_{N-1}, p\right) \\
& \geq-R\|x\|-R \sum_{i=1}^{N-1}\left\|p_{i}\right\|-R\|p\|-R \sum_{i=1}^{N-1}\left\|x_{i}\right\|-(N-1) R^{2} \\
& \geq-R\|x\|-R \sum_{i=1}^{N-1}\left\|p_{i}\right\|-(2 N-1) R^{2} .
\end{aligned}
$$

Therefore $\left|L_{H}^{* *}\left(x, p_{1}, \ldots, p_{N-1}\right)\right| \leq R\|x\|+R \sum_{i=1}^{N-1}\left\|p_{i}\right\|+(2 N-1) R^{2}$. The estimate for $H_{L_{H}^{* *}}$ can be easily deduced from its definition together with the estimate on $L_{H}^{* *}$. This completes the proof of part (1). 
For (2) set $D=\Omega \times \Pi_{i=1}^{N-1} B_{R}$, then $D \subset \tilde{B}_{N R}$ where $\tilde{B}_{N R}$ is a ball with radius $N R$ in $\mathbb{R}^{d N}$. Now assuming $f=L_{H}^{*}$ in Lemma 3.3, we have that $\tilde{f}=L_{H}^{* *}$. Therefore $L_{H}^{* *}$ is Lipschitz in $\left(\mathbb{R}^{d}\right)^{N}$ with $\operatorname{Lip}\left(L_{H}^{* *}\right) \leq N R$. To prove that $H_{L_{H}^{* *}}$ is Lipschitz continuous, we first fix $y \in \mathbb{R}^{d}$ and define $f_{y}:\left(\mathbb{R}^{d}\right)^{N-1} \rightarrow \mathbb{R}$ by

$$
f_{y}\left(p_{1}, \ldots, p_{N-1}\right)=L_{H}^{* *}\left(y, p_{1}, \ldots p_{N-1}\right) .
$$

Assuming $D=B_{R} \subset \mathbb{R}^{N}$ in Proposition 3.3, we obtain that the map

$$
\left(x_{1}, \ldots, x_{N-1}\right) \rightarrow \tilde{f}_{y}\left(x_{1}, \ldots, x_{N-1}\right)=H_{L_{H}^{* *}}\left(y, x_{1}, \ldots, x_{N-1}\right)
$$

is Lipschitz and

$$
\left|H_{L_{H}^{* *}}\left(y, x_{1}, \ldots, x_{N-1}\right)-H_{L_{H}^{* *}}\left(y, z_{1}, \ldots, z_{N-1}\right)\right| \leq R \sum_{i=1}^{N-1}\left\|x_{i}-z_{i}\right\|
$$

for all $\left(x_{i}\right),\left(z_{i}\right) \in\left(\mathbb{R}^{d}\right)^{N-1}$. Noticing that the Lipschitz constant $R$ is independent of $y$, the above inequality holds for all $\left(x_{i}\right),\left(z_{i}\right) \in\left(\mathbb{R}^{d}\right)^{N-1}$ and $y \in \mathbb{R}^{d}$. To prove $H_{L_{H}^{* *}}\left(y, x_{1}, \ldots, x_{N-1}\right)$ is Lipschitz with respect to the first variable $y$, let $r>0$ and $y_{1}, y_{2} \in \mathbb{R}^{d}$. Let $p_{1}, \ldots, p_{N-1}$ and $q_{1}, \ldots, q_{N-1}$ be such that

$$
\sum_{i=1}^{N-1}\left\langle x_{i}, q_{i}\right\rangle-L_{H}^{* *}\left(y_{1}, q_{1}, \ldots, q_{N-1}\right) \leq H_{L_{H}^{* *}}\left(y_{1}, x_{1}, \ldots, x_{N-1}\right) \leq \sum_{i=1}^{N-1}\left\langle x_{i}, p_{i}\right\rangle-L_{H}^{* *}\left(y_{1}, p_{1}, \ldots, p_{N-1}\right)+r
$$

and

$$
\sum_{i=1}^{N-1}\left\langle x_{i}, p_{i}\right\rangle-L_{H}^{* *}\left(y_{2}, p_{1}, \ldots, p_{N-1}\right) \leq H_{L_{H}^{* *}}\left(y_{2}, x_{1}, \ldots, x_{N-1}\right) \leq \sum_{i=1}^{N-1}\left\langle x_{i}, q_{i}\right\rangle-L_{H}^{* *}\left(y_{2}, q_{1}, \ldots, q_{N-1}\right)+r .
$$

It follows that

$$
\begin{aligned}
L_{H}^{* *}\left(y_{2}, q_{1}, \ldots, q_{N-1}\right)-L_{H}^{* *}\left(y_{1}, q_{1}, \ldots, q_{N-1}\right)-r & \leq H_{L_{H}^{* *}}\left(y_{1}, x_{1}, \ldots, x_{N-1}\right)-H_{L_{H}^{* *}}\left(y_{2}, x_{1}, \ldots, x_{N-1}\right) \\
& \leq L_{H}^{* *}\left(y_{2}, p_{1}, \ldots, p_{N-1}\right)-L_{H}^{* *}\left(y_{1}, p_{1}, \ldots, p_{N-1}\right)+r .
\end{aligned}
$$

Since $L_{H}^{* *}$ is Lipschitz,

$$
-N R\left\|y_{1}-y_{2}\right\|-r \leq H_{L_{H}^{* *}}\left(y_{1}, x_{1}, \ldots, x_{N-1}\right)-H_{L_{H}^{* *}}\left(y_{2}, x_{1}, \ldots, x_{N-1}\right) \leq N R\left\|y_{1}-y_{2}\right\|+r .
$$

Since $r>0$ is arbitrary we obtain

$$
-N R\left\|y_{1}-y_{2}\right\| \leq H_{L_{H}^{* *}}\left(y_{1}, x_{1}, \ldots, x_{N-1}\right)-H_{L_{H}^{* *}}\left(y_{2}, x_{1}, \ldots, x_{N-1}\right) \leq N R\left\|y_{1}-y_{2}\right\| .
$$

This together with (47) prove that $H_{L_{H}^{* *}}$ is Lipschitz continuous and that $\operatorname{Lip}\left(H_{L_{H}^{* *}}\right) \leq N R$.

Proof of Proposition 3.1, 1) By Lemma 3.2, we have that $H_{r e g}^{1}:=H_{L_{H}^{* *}}$ is a concave-convex Hamiltonian on $\mathbb{R}^{d} \times\left(\mathbb{R}^{d}\right)^{N-1}$ whose restriction to $\bar{\Omega}^{N}$ is $N$-sub-antisymmetric, hence belong to $\mathcal{H}_{N}^{-}(\Omega)$.

2) To show that $H_{r e g}^{2}$ is $N$-antisymmetric note that

$$
N H_{r e g}^{2}(\mathbf{x})=(N-1) H_{r e g}^{1}(\mathbf{x})-\sum_{i=1}^{N-1} H_{r e g}^{1}\left(\sigma^{i}(\mathbf{x})\right)=\sum_{i=1}^{N-1}\left[H_{r e g}^{1}(\mathbf{x})-H_{r e g}^{1}\left(\sigma^{i}(\mathbf{x})\right)\right]
$$

and each of the terms $H_{r e g}^{1}(\mathbf{x})-H_{r e g}^{1}\left(\sigma^{i}(\mathbf{x})\right)$ is easily seen to be $N$-antisymmetric.

Now $H_{r e g}^{2}$ dominates $H_{r e g}^{1}$ since

$$
N\left[H_{r e g}^{2}(\mathbf{x})-H_{r e g}^{1}(\mathbf{x})\right]=-H_{r e g}^{1}(\mathbf{x})-\sum_{i=1}^{N-1} H_{r e g}^{1}\left(R^{i}(\mathbf{x})\right) \geq 0,
$$

since $H_{r e g}^{1}$ is $N$-sub-antisymmetric. 
3) For $x \in \Omega$ and $p_{1}, \ldots, p_{N-1} \in B_{R}$ we have

$$
\begin{aligned}
L_{H_{r e g}^{1}}\left(x, p_{1}, \ldots, p_{N-1}\right) & =\sup _{y_{i} \in \Omega}\left\{\sum_{i=1}^{N-1}\left\langle p_{i}, y_{i}\right\rangle-H_{L_{H}^{* *}}\left(x, y_{1}, \ldots, y_{N-1}\right)\right\} \\
& =\sup _{y_{i} \in \Omega}\left\{\sum_{i=1}^{N-1}\left\langle p_{i}, y_{i}\right\rangle-\sup _{q_{i} \in B_{R}}\left\{\sum_{i=1}^{N-1}\left\langle q_{i}, y_{i}\right\rangle-L_{H}^{* *}\left(x, q_{1}, \ldots, q_{N-1}\right)\right\}\right\} \\
& \left.=\sup _{y_{i} \in \Omega} \inf _{q_{i} \in B_{R}}\left\{\sum_{i=1}^{N-1}\left\langle p_{i}, y_{i}\right\rangle-\sum_{i=1}^{N-1}\left\langle q_{i}, y_{i}\right\rangle+L_{H}^{* *}\left(x, q_{1}, \ldots, q_{N-1}\right)\right\}\right\} \\
& \left.\leq \inf _{q_{i} \in B_{R}} \sup _{y_{i} \in \Omega}\left\{\sum_{i=1}^{N-1}\left\langle p_{i}, y_{i}\right\rangle-\sum_{i=1}^{N-1}\left\langle q_{i}, y_{i}\right\rangle+L_{H}^{* *}\left(x, q_{1}, \ldots, q_{N-1}\right)\right\}\right\} \\
& \left.\leq \sup _{y_{i} \in \Omega}\left\{\sum_{i=1}^{N-1}\left\langle p_{i}, y_{i}\right\rangle-\sum_{i=1}^{N-1}\left\langle p_{i}, y_{i}\right\rangle+L_{H}^{* *}\left(x, p_{1}, \ldots, p_{N-1}\right)\right\}\right\} \\
& =L_{H}^{* *}\left(x, p_{1}, \ldots, p_{N-1}\right)
\end{aligned}
$$

On the other hand by Lemma (3.1) we have $L_{H}^{* *} \leq L_{H}$, and therefore $L_{H_{r e g}^{1}} \leq L_{H}$. It also follows from part 2) that $L_{H_{r e g}^{2}} \leq L_{H_{r e g}^{1}}$. This completes the proof of part 3).

Parts 4), 5) and 6) are the subject of the preceding Lemmas.

\section{Proof of Theorem 1.6: Existence}

We first show that the minimization problem $\left(\mathrm{DK}_{\text {sym }}^{2}\right)$ is attained. Let $B_{R}$ be a ball such that $u_{i}(\bar{\Omega}) \subset B_{R}$ for all $i=1, \ldots, N-1$. Let $\left\{H_{n}\right\}$ be a sequence in $\mathcal{H}_{N}(\Omega)$ such that $L_{H_{n}}$ is a minimizing sequence for $\left(\mathrm{DK}_{\text {sym }}^{2}\right)$. Denoting $H_{n}^{1}:=\left(H_{n}\right)_{\text {reg }}^{1}$, we get from Proposition 3.1 that $L_{H_{n}^{1}} \leq L_{H_{n}}$ on $\bar{\Omega} \times B_{R}^{N-1}$ and therefore $L_{H_{n}^{1}}$ is also minimizing for $\left(\mathrm{DK}_{\text {sym }}^{2}\right)$. It also follows from Proposition 3.1 that $L_{H_{n}^{1}}$ and $H_{n}^{1}$, are uniformly Lipschitz with $\operatorname{Lip}\left(H_{n}^{1}\right), \operatorname{Lip}\left(L_{H_{n}^{1}}\right) \leq N R$. Moreover,

$$
\left|H_{n}^{1}\left(x, y_{1}, \ldots, y_{N-1}\right)\right| \leq R\|x\|+R \sum_{i=1}^{N-1}\left\|y_{i}\right\|+2 N R^{2} \text { for all } x \text { and }\left(y_{i}\right)_{i=1}^{N-1} \text { in } \mathbb{R}^{d},
$$

and

$$
\left|L_{H_{n}^{1}}\left(x, p_{1}, \ldots, p_{N-1}\right)\right| \leq R\|x\|+R \sum_{i=1}^{N-1}\left\|p_{i}\right\|+(2 N-1) R^{2} \text { for all } x, p_{1}, \ldots, p_{N-1} \text { in } \mathbb{R}^{d} .
$$

By Arzela-Ascoli's theorem, there exists two Lipschitz functions $\tilde{H}$ and $\tilde{L}: \mathbb{R}^{d} \times \mathbb{R}^{d(N-1)} \rightarrow \mathbb{R}$ such that $H_{n}^{1}$ converges to $\tilde{H}$ and $L_{n}^{1}$ converges to $\tilde{L}$ uniformly on every compact set of $\mathbb{R}^{N} \times \ldots \times \mathbb{R}^{N}$. This implies that $\tilde{H} \in \mathcal{H}_{N}^{-}(\Omega)$. Note that

$$
L_{H_{n}^{1}}\left(x, p_{1}, \ldots, p_{N-1}\right)+H_{n}^{1}\left(x, y_{1}, \ldots, y_{N-1}\right) \geq \sum_{i=1}^{N-1}\left\langle y_{i}, p_{i}\right\rangle,
$$

for all $x, p_{1}, \ldots, p_{N-1} \in \mathbb{R}^{N}$ and $y_{1}, \ldots, y_{N-1} \in \bar{\Omega}$, from which we have

$$
\tilde{L}\left(x, p_{1}, \ldots, p_{N-1}\right) \geq \sum_{i=1}^{N-1}\left\langle y_{i}, p_{i}\right\rangle-\tilde{H}\left(x, y_{1}, \ldots, y_{N-1}\right),
$$

for all $x, p_{1}, \ldots, p_{N-1} \in \mathbb{R}^{N}$ and $y_{1}, \ldots, y_{N-1} \in \bar{\Omega}$. This implies that $L_{\tilde{H}} \leq \tilde{L}$. Let $H_{\infty}^{1}=\tilde{H}_{r e g}^{1}$ and $H_{\infty}^{2}=\tilde{H}_{r e g}^{2}$ be the regularizations of $\tilde{H}$ defined in the previous section. Set $L_{\infty}^{i}=L_{H_{\infty}^{i}}$ for $i=1,2$. It follows from 
Proposition 3.1 that $L_{H_{\infty}^{2}} \leq L_{H_{\infty}^{1}} \leq L_{\tilde{H}}$ on $\bar{\Omega} \times B_{R}^{N-1}$, from which we have

$$
\begin{aligned}
\mathrm{DK}_{\text {sym }}^{2} & =\int_{\Omega} L_{\tilde{H}}\left(x, u_{1}(x), \ldots, u_{N-1}(x)\right) d \mu \\
& =\int_{\Omega} L_{\infty}^{2}\left(x, u_{1}(x), \ldots, u_{N-1}(x)\right) d \mu \\
& =\int_{\Omega} L_{\infty}^{1}\left(x, u_{1}(x), \ldots, u_{N-1}(x)\right) d \mu .
\end{aligned}
$$

For the rest of the proof, we shall need the following two technical lemmas. The first one relates $L_{H}^{*}$ to the standard Legendre transform of $H$ (extended beyond $\Omega^{N}$ to the whole of $\mathbb{R}^{d N}$ ).

Lemma 4.1 Let $H_{\infty}=H_{\infty}^{1}$ be the concave-convex Hamiltonian obtained above and $L_{\infty}=L_{\infty}^{1}$. For each $x \in \bar{\Omega}$, define $f_{x}:\left(\mathbb{R}^{d}\right)^{N-1} \rightarrow \mathbb{R}$ by

$$
f_{x}\left(y_{1}, \ldots, y_{N-1}\right):=H_{\infty}\left(x, y_{1}, \ldots, y_{N-1}\right) .
$$

We also define $\tilde{f}_{x}:\left(\mathbb{R}^{d}\right)^{N-1} \rightarrow \mathbb{R} \cup\{+\infty\}$ by

$$
\tilde{f}_{x}\left(y_{1}, \ldots, y_{N-1}\right):=f_{x}\left(y_{1}, \ldots, y_{N-1}\right) \text { if } y_{1}, \ldots, y_{N-1} \in \bar{\Omega}^{N-1} \text { and }+\infty \text { otherwise. }
$$

Let $\left(\tilde{f}_{x}\right)^{*}$ be the standard Fenchel dual of $\tilde{f}_{x}$ on $\left(\mathbb{R}^{d}\right)^{N-1}$ in such a way that $\left(\tilde{f}_{x}\right)^{* * *}=\left(\tilde{f}_{x}\right)^{*}$ on $\left(\mathbb{R}^{d}\right)^{N-1}$. We then have,

$$
f_{x}=\left(\tilde{f}_{x}\right)^{* *}=\tilde{f}_{x} \text { on } \bar{\Omega}^{N-1}
$$

and

$$
\begin{aligned}
L_{\infty}\left(x, p_{1}, \ldots, p_{N-1}\right) & =\sup _{\left(z_{i}\right) \in \bar{\Omega}^{N-1}}\left\{\sum_{i=1}^{N-1}\left\langle z_{i}, p_{i}\right\rangle-\left(\tilde{f}_{x}\right)^{* *}\left(z_{1}, \ldots, z_{N-1}\right)\right\} \\
& =\sup _{\left(z_{i}\right) \in\left(\mathbb{R}^{d}\right)^{N-1}}\left\{\sum_{i=1}^{N-1}\left\langle z_{i}, p_{i}\right\rangle-\left(\tilde{f}_{x}\right)^{* *}\left(z_{1}, \ldots, z_{N-1}\right)\right\} .
\end{aligned}
$$

Proof. Since $\left(\tilde{f}_{x}\right)^{* *}$ is the largest convex function below $\tilde{f}_{x}$ we have and $f_{x} \leq\left(\tilde{f}_{x}\right)^{* *} \leq \tilde{f}_{x}$, from which we obtain $f_{x}=\left(\tilde{f}_{x}\right)^{* *}=\tilde{f}_{x}$ on $\bar{\Omega}^{N-1}$.

For (49), we first deduce from (48) that

$$
\begin{aligned}
\left(\tilde{f}_{x}\right)^{*}\left(y_{1}, \ldots, y_{N-1}\right) & =\left(\tilde{f}_{x}\right)^{* * *}\left(y_{1}, \ldots, y_{N-1}\right) \\
& =\sup _{z \in \mathbb{R}^{d(N-1}}\left\{\sum_{i=1}^{N-1}\left\langle z_{i}, y_{i}\right\rangle-\left(\tilde{f}_{x}\right)^{* *}\left(z_{1}, \ldots, z_{N-1}\right)\right\} \\
& \geq \sup _{z \in B_{R}^{N-1}}\left\{\sum_{i=1}^{N-1}\left\langle z_{i}, y_{i}\right\rangle-\left(\tilde{f}_{x}\right)^{* *}\left(z_{1}, \ldots, z_{N-1}\right)\right\} \\
& \geq \sup _{z \in \Omega^{N-1}}\left\{\sum_{i=1}^{N-1}\left\langle z_{i}, y_{i}\right\rangle-\left(\tilde{f}_{x}\right)^{* *}\left(z_{1}, \ldots, z_{N-1}\right)\right\} \\
& =\sup _{z \in \Omega^{N-1}}\left\{\sum_{i=1}^{N-1}\left\langle z_{i}, y_{i}\right\rangle-f_{x}\left(z_{1}, \ldots, z_{N-1}\right)\right\} \\
& =\sup _{z \in \Omega^{N-1}}\left\{\sum_{i=1}^{N-1}\left\langle z_{i}, y_{i}\right\rangle-\tilde{f}_{x}\left(z_{1}, \ldots, z_{N-1}\right)\right\} \\
& =\left(\tilde{f}_{x}\right)^{*}\left(y_{1}, \ldots, y_{N-1}\right),
\end{aligned}
$$


from which we have the desired result.

Fix now $H_{\infty}$ as above and let $H \in C\left(\bar{\Omega}^{N}\right)$. For each $\lambda>0$ and $r \in(-1,1)$, we associate the following three functionals.

$$
\begin{aligned}
L_{r, \lambda}\left(x, p_{1}, \ldots, p_{N-1}\right):=\sup _{\left(z_{i}\right) \in \bar{\Omega}^{N-1}}\left\{\begin{array}{l}
\sum_{i=1}^{N-1}\left\langle z_{i}, p_{i}\right\rangle-\left(\tilde{f}_{x}\right)^{* *}\left(z_{1}, \ldots, z_{N-1}\right)-\frac{\lambda}{2}\left[\sum_{i=1}^{N-1}\left\|z_{i}\right\|^{2}-(N-1)\|x\|^{2}\right] . \\
\left.+r H\left(x, z_{1}, \ldots, z_{N-1}\right)\right\}
\end{array}\right. \\
L_{\lambda}\left(x, p_{1}, \ldots, p_{N-1}\right):=\sup _{\left(z_{i}\right) \in \mathbb{R}^{d(N-1)}}\left\{\sum_{i=1}^{N-1}\left\langle z_{i}, p_{i}\right\rangle-\left(\tilde{f}_{x}\right)^{* *}\left(z_{1}, \ldots, z_{N-1}\right)-\frac{\lambda}{2}\left[\sum_{i=1}^{N-1}\left\|z_{i}\right\|^{2}-(N-1)\|x\|^{2}\right]\right\} \\
L_{r}\left(x, p_{1}, \ldots, p_{N-1}\right):=\sup _{\left(z_{i}\right) \in \bar{\Omega}^{N-1}}\left\{\sum_{i=1}^{N-1}\left\langle z_{i}, p_{i}\right\rangle-H_{\infty}\left(x, z_{1}, \ldots, z_{N-1}\right)+r H\left(x, z_{1}, \ldots, z_{N-1}\right)\right\} .
\end{aligned}
$$

Lemma 4.2 Let $H \in C\left(\bar{\Omega}^{N}\right)$ be such that $H_{\infty}-r H \in \mathcal{H}_{N}^{-}(\Omega)$ for all $r \in(-1,1)$. Then, the following hold:

1. For every $\left(x, p_{1}, \ldots, p_{N-1}\right) \in \mathbb{R}^{d} \times \mathbb{R}^{d(N-1)}$, we have

$$
\lim _{\lambda \rightarrow 0^{+}} L_{\lambda}\left(x, p_{1}, \ldots, p_{N-1}\right)=L_{\infty}\left(x, p_{1}, \ldots, p_{N-1}\right) \text { and } \lim _{\lambda \rightarrow 0^{+}} L_{r, \lambda}\left(x, p_{1}, \ldots, p_{N-1}\right)=L_{r}\left(x, p_{1}, \ldots, p_{N-1}\right) .
$$

2. For all $x \in \mathbb{R}^{d}$, the function $\left(p_{1}, \ldots, p_{N-1}\right) \rightarrow L_{\lambda}\left(x, p_{1}, \ldots, p_{N-1}\right)$ is differentiable.

3. For every $\left(x, p_{1}, \ldots, p_{N-1}\right) \in \mathbb{R}^{d} \times \mathbb{R}^{d(N-1)}$, we have

$$
\lim _{r \rightarrow 0} \frac{L_{r, \lambda}\left(x, p_{1}, \ldots, p_{N-1}\right)-L_{\lambda}\left(x, p_{1}, \ldots, p_{N-1}\right)}{r}=H\left(\nabla_{2, \ldots, N} L_{\lambda}\left(x, p_{1}, \ldots, p_{N-1}\right), x\right) .
$$

Proof. Yosida's regularization of convex functions and Lemma 4.1 yield that

$$
\begin{aligned}
\lim _{\lambda \rightarrow 0^{+}} L_{r, \lambda}\left(x, p_{1}, \ldots, p_{N-1}\right) & =\sup _{\left(z_{i}\right) \in \bar{\Omega}^{N-1}}\left\{\sum_{i=1}^{N-1}\left\langle z_{i}, p_{i}\right\rangle-\left(\tilde{f}_{x}\right)^{* *}\left(z_{1}, \ldots, z_{N-1}\right)-r H\left(x, z_{1}, \ldots, z_{N-1}\right)\right\} \\
& =\sup _{\left(z_{i}\right) \in \bar{\Omega}^{N-1}}\left\{\sum_{i=1}^{N-1}\left\langle z_{i}, p_{i}\right\rangle-H_{\infty}\left(x, z_{1}, \ldots, z_{N-1}\right)-r H\left(x, z_{1}, \ldots, z_{N-1}\right)\right\} \\
= & L_{r}\left(x, p_{1}, \ldots, p_{N-1}\right) .
\end{aligned}
$$

We also have

$$
\lim _{\lambda \rightarrow 0} L_{\lambda}\left(x, p_{1}, \ldots, p_{N-1}\right)=\sup _{\left(z_{i}\right) \in \mathbb{R}^{d(N-1)}}\left\{\sum_{i=1}^{N-1}\left\langle z_{i}, p_{i}\right\rangle-\left(\tilde{f}_{x}\right)^{* *}\left(z_{1}, \ldots, z_{N-1}\right)\right\},
$$

which, together with Lemma 4.1, yield that $\lim _{\lambda \rightarrow 0} L_{\lambda}\left(x, p_{1}, \ldots, p_{N-1}\right)=L_{\infty}\left(x, p_{1}, \ldots, p_{N-1}\right)$.

(2) follows from the fact that the Yosida regularization of convex functions are differentiable.

(3) We let $z_{(r, \lambda, i)} \in \bar{\Omega}$ and $z_{(r, \lambda, i)}^{\prime} \in \mathbb{R}^{d}$ be such that

$$
\begin{aligned}
L_{r, \lambda}\left(x, p_{1}, \ldots, p_{N-1}\right) \leq & \sum_{i=1}^{N-1}\left\langle z_{(r, \lambda, i)}, p_{i}\right\rangle-\left(\tilde{f}_{x}\right)^{* *}\left(z_{(r, \lambda, 1)}, \ldots, z_{(r, \lambda, N-1)}\right)-\frac{\lambda}{2} \sum_{i=1}^{N-1}\left\|z_{(r, \lambda, i)}\right\|^{2} \\
& +\lambda \frac{(N-1)\|x\|^{2}}{2}+r H\left(x, z_{(r, \lambda, 1)}, \ldots, z_{(r, \lambda, N-1)}\right)+r^{2}, \\
L_{\lambda}\left(x, p_{1}, \ldots, p_{N-1}\right) \leq & \sum_{i=1}^{N-1}\left\langle z_{\lambda, i}^{\prime}, p_{i}\right\rangle-\left(\tilde{f}_{x}\right)^{* *}\left(z_{(r, \lambda, 1)}^{\prime}, \ldots, z_{(r, \lambda, N-1)}\right)-\frac{\lambda}{2} \sum_{i=1}^{N-1}\left\|z_{(r, \lambda, i)}^{\prime}\right\|^{2}+\lambda \frac{(N-1)\|x\|^{2}}{2}+r^{2} .
\end{aligned}
$$


Therefore,

$$
\begin{aligned}
r H\left(x, z_{(r, \lambda, 1)}^{\prime}, \ldots, z_{(r, \lambda, N-1)}^{\prime}\right)-r^{2} & \leq L_{r, \lambda}\left(x, p_{1}, \ldots, p_{N-1}\right)-L_{\lambda}\left(x, p_{1}, \ldots, p_{N-1}\right) \\
& \leq r H\left(x, z_{(r, \lambda, 1)}, \ldots, z_{(r, \lambda, N-1)}\right)+r^{2}
\end{aligned}
$$

By the definition of $L_{\lambda}$, we have $\sup _{r \in[-1,1]}\left\|z_{r, \lambda, i}^{\prime}\right\|<\infty$. Suppose now that, up to a subsequence, $z_{r, \lambda, i} \rightarrow$ $z_{i} \in \bar{\Omega}$ and $z_{r, \lambda, i}^{\prime} \rightarrow z_{\lambda, i}^{\prime}$ as $r \rightarrow 0$. This together with the definition of $L_{r, \lambda}$ and $L_{\lambda}$ imply that

$$
\begin{aligned}
L_{\lambda}\left(x, p_{1}, \ldots, p_{N-1}\right) & =\sum_{i=1}^{N-1}\left\langle z_{(\lambda, i)}, p_{i}\right\rangle-\left(\tilde{f}_{x}\right)^{* *}\left(z_{(\lambda, 1)}, \ldots, z_{(\lambda, N-1)}\right)-\frac{\lambda}{2} \sum_{i=1}^{N-1}\left\|z_{(\lambda, i)}\right\|^{2}+\lambda(N-1) \frac{\|x\|^{2}}{2} \\
& =\sum_{i=1}^{N-1}\left\langle z_{\lambda, i}^{\prime}, p_{i}\right\rangle-\left(\tilde{f}_{x}\right)^{* *}\left(z_{(\lambda, 1)}, \ldots, z_{(\lambda, N-1)}^{\prime}\right)-\frac{\lambda}{2} \sum_{i=1}^{N-1}\left\|z_{(\lambda, i)}^{\prime}\right\|^{2}+\lambda(N-1) \frac{\|x\|^{2}}{2}
\end{aligned}
$$

from which we obtain that

$$
z_{\lambda, i}=z_{\lambda, i}^{\prime}=\nabla_{i} L_{\lambda}\left(x, p_{1}, \ldots, p_{N-1}\right) \in \bar{\Omega}, \quad i=2, \ldots, N
$$

It then follows from (50) that

$$
\lim _{r \rightarrow 0} \frac{L_{r, \lambda}\left(x, p_{1}, \ldots, p_{N-1}\right)-L_{\lambda}\left(x, p_{1}, \ldots, p_{N-1}\right)}{r}=H\left(\nabla_{2, \ldots, N} L_{\lambda}\left(x, p_{1}, \ldots, p_{N-1}\right), x\right) .
$$

End of the proof of Theorem 1.6 (Existence): For each $\lambda>0, x \in \bar{\Omega}$ and $p \in \mathbb{R}^{N}$, we define

$$
\bar{S}_{\lambda, i}\left(x, p_{1}, \ldots, p_{N-1}\right)=\nabla_{i} L_{\lambda}\left(x, p_{1}, \ldots, p_{N-1}\right) \quad i=2, \ldots, N
$$

We have that $\bar{S}_{\lambda, i}\left(x, p_{1}, \ldots, p_{N-1}\right) \rightarrow \bar{S}_{0, i}\left(x, p_{1}, \ldots, p_{N-1}\right)$ where $\bar{S}_{0, i}\left(x, p_{1}, \ldots, p_{N-1}\right)$ is the unique element with minimal norm in $\partial_{i} L_{\infty}\left(x, p_{1}, \ldots, p_{N-1}\right)$.

Set $S_{\lambda, i}(x)=\bar{S}_{\lambda, i}\left(x, u_{1}(x), \ldots, u_{N-1}(x)\right)$ and $S_{i}(x)=S_{0, i}\left(x, u_{1}(x), \ldots, u_{N-1}(x)\right)$. For each $r>0, \lambda \in[0,1]$ and $x \in \bar{\Omega}$, define

$$
\eta_{r}(\lambda, x)=\frac{L_{r, \lambda}\left(x, u_{1}(x), \ldots, u_{N-1} x\right)-L_{\lambda}\left(x, u_{1}(x), \ldots, u_{N-1}(x)\right)}{r} .
$$

Note that the function $r \rightarrow L_{r, \lambda}\left(x, u_{1}(x), \ldots, u_{N-1}(x)\right)$ is a convex function because it is supremum of a family of linear functions. Thus, for fixed $(x, \lambda) \in \Omega \times[0,1]$, the function $r \rightarrow \eta_{r}(\lambda, x)$ is non-decreasing. Setting $\eta_{0}(\lambda, x)$ to be $H\left(x, S_{\lambda, 1}(x), \ldots, S_{\lambda, N-1}(x)\right)$ for $\lambda>0$ and $\eta_{0}(0, x)=H\left(x, S_{1}(x), \ldots, S_{N-1}(x)\right)$, we have that both functions $\lambda \rightarrow \eta_{r}(\lambda, x)$ and $\lambda \rightarrow \eta_{0}(\lambda, x)$ are continuous. It follows from Dini's Theorem, that for a fixed $x, \eta_{r}(\lambda, x)$ converges uniformly to $\eta_{0}(\lambda, x)$ as $r \rightarrow 0$ with respect to $\lambda \in[0,1]$. Note also that thanks to (51) we have that $S_{\lambda, i}, S_{i}: \bar{\Omega} \rightarrow \bar{\Omega}$ and for all $x \in \Omega$.

$$
\left(S_{1} x, \ldots, S_{N-1} x\right) \in \partial_{2, \ldots, N} L_{\infty}\left(x, u_{1}(x), \ldots, u_{N-1}(x)\right) .
$$

We now show that

$$
\int_{\Omega} H\left(x, S_{1} x, \ldots, S_{N-1} x\right) d \mu=0 \text { for all } H \in C\left(\bar{\Omega}^{N}\right) \text { with } H_{\infty}-r H \in \mathcal{H}_{N}^{-}(\Omega), r \in(-1,1) .
$$

Indeed, since $\left|H\left(x, S_{1} x, \ldots, S_{N-1} x\right)\right| \leq\|H\|_{L^{\infty}\left(\bar{\Omega}^{N}\right)}$, we get from Lebesgue's dominated convergence Theorem,

$$
\lim _{\lambda \rightarrow 0} \int_{\Omega} H\left(x, S_{\lambda, 1}(x), \ldots, S_{\lambda, N-1}(x)\right) d \mu(x)=\int_{\Omega} H\left(x, S_{1} x, \ldots, S_{N-1} x\right) d \mu(x) .
$$

From (50) we have

$$
\left|\frac{L_{r, \lambda}\left(x, p_{1}, \ldots, p_{N-1}\right)-L_{\lambda}\left(x, p_{1}, \ldots, p_{N-1}\right)}{r}\right| \leq\|H\|_{L^{\infty}\left(\bar{\Omega}^{N}\right)}+|r|,
$$


from which follows that

$$
\begin{aligned}
\int_{\Omega} H\left(x, S_{1} x, \ldots, S_{N-1} x\right) d \mu(x) & =\int_{\Omega} \lim _{\lambda \rightarrow 0} \lim _{r \rightarrow 0^{+}} \frac{L_{r, \lambda}\left(x, u_{1}(x), \ldots, u_{N-1}(x)\right)-L_{\lambda}\left(x, u_{1}(x), \ldots, u_{N-1}(x)\right)}{r} d \mu \\
& =\int_{\Omega} \lim _{\lambda \rightarrow 0} \lim _{r \rightarrow 0^{+}} \eta_{r}(\lambda, x) d \mu \\
& =\int_{\Omega} \lim _{r \rightarrow 0^{+}} \lim _{\lambda \rightarrow 0} \eta_{r}(\lambda, x) d \mu \quad \text { (due to the uniform convergence) } \\
& =\int_{\Omega} \lim _{r \rightarrow 0^{+}} \eta_{r}(0, x) d \mu \\
& =\lim _{r \rightarrow 0^{+}} \int_{\Omega} \eta_{r}(0, x) d \mu \quad \text { (due to the monotone convergence theorem) } \\
& =\lim _{r \rightarrow 0^{+}} \int_{\Omega} \frac{L_{r}\left(x, u_{1}(x), \ldots, u_{N-1}(x)\right)-L_{\infty}\left(x, u_{1}(x), \ldots, u_{N-1}(x)\right)}{r} d \mu \\
& \left.\geq 0, \quad \text { in view of the optimality of } H_{\infty} \text { compared to } H_{\infty}-r H\right) .
\end{aligned}
$$

In other words, we have $\int_{\Omega} H\left(x, S_{1} x, \ldots, S_{N-1} x\right) d \mu \geq 0$. By the same argument considering $r \rightarrow 0^{-}$, one has $\int_{\Omega} H\left(x, S_{1} x, \ldots, S_{N-1} x\right) d \mu \leq 0$ and therefore the latter is indeed zero as desired.

Note now that (53) yields that

$$
\int_{\Omega} H_{\infty}\left(x, S_{1} x, \ldots, S_{N-1} x\right) d \mu=0
$$

and

$$
\int_{\Omega} H\left(x, S_{1} x, \ldots, S_{N-1} x\right) d \mu=0 \text { for all } H \in \mathcal{H}_{N}(\Omega) .
$$

It follows from Lemma 2.3 that $S$ is measure preserving, that $S_{i}=S_{1}^{i}$ and that $S_{1}^{N}=I$. We shall now write $S$ for $S_{1}$.

In order to show that $\mathrm{DK}_{\mathrm{sym}}^{2}=\mathrm{MK}_{\mathrm{sym}}$, we note that clearly $\mathrm{MK}_{\mathrm{sym}} \leq \mathrm{DK}_{\mathrm{sym}}^{2}$. For the reverse inequality, we use the fact that $\left(S x, \ldots, S^{N-1} x\right) \in \partial_{2, \ldots, N} L_{\infty}\left(x, u_{1}(x), \ldots, u_{N-1}(x)\right)$ together with $\left(\tilde{f}_{x}\right)^{* *}$ being the Fenchel dual of $L$ with respect to the last $N-1$ variables and Lemma 4.1 to obtain that

$$
\left(u_{1}(x), \ldots, u_{N-1}(x)\right) \in \partial\left(\tilde{f}_{x}\right)^{* *}\left(S x, \ldots, S^{N-1} x\right) .
$$

Since meas $(\partial \Omega)=0$, the set $\cup_{i=1}^{N-1} S^{-i}(\partial \Omega)$ is negligible and for each $x \in \Omega \backslash \cup_{i=1}^{N-1} S^{-i}(\partial \Omega)$, one has

$$
\partial\left(\tilde{f}_{x}\right)^{* *}\left(S x, \ldots, S^{N-1} x\right)=\partial_{2, \ldots, N} H_{\infty}\left(x, S x, \ldots, S^{N-1} x\right) .
$$

It follows that

$$
\left(u_{1}(x), \ldots, u_{N-1}(x)\right) \in \partial_{2, \ldots, N} H_{\infty}\left(x, S x, \ldots, S^{N-1} x\right) \quad \text { a.e. } \quad x \in \Omega .
$$

We finally get that

$$
\begin{aligned}
\mathrm{DK}_{\mathrm{sym}}^{2} & =\int_{\Omega} L_{\infty}\left(x, u_{1}(x), \ldots, u_{N-1}(x)\right) d \mu(x) \\
& =\int_{\Omega} L_{\infty}\left(x, u_{1}(x), \ldots, u_{N-1}(x)\right) d \mu(x)+\int_{\Omega} H_{\infty}\left(x, S x, \ldots, S^{N-1} x\right) d \mu(x) \\
& =\int_{\Omega} L_{\infty}\left(x, u_{1}(x), \ldots, u_{N-1}(x)\right) d \mu(x)+\int_{\Omega}\left(\tilde{f}_{x}\right)^{* *}\left(S x, \ldots, S^{N-1} x\right) d \mu(x) \\
& =\int_{\Omega} \sum_{i=1}^{N-1}\left\langle u_{i}(x), S^{i}(x)\right\rangle d \mu(x) \leq \mathrm{MK}_{\mathrm{sym}} .
\end{aligned}
$$

If now $u_{i} \in W_{l o c}^{1,1}(\Omega)$ for $i=1,2, \ldots, N-1$, or if $S$ is a.e. differentiable, then by Theorem 7.1 of the Appendix, there exists a full measure subset $\Omega_{0}$ of $\Omega$ that $\nabla_{2, \ldots, N} H_{\infty}\left(x, S x, \ldots, S^{N-1} x\right)$ exists for all $x \in \Omega_{0}$. It follows that

$$
\left(u_{1}(x), \ldots, u_{N-1}(x)\right)=\nabla_{2, \ldots, N} H_{\infty}\left(x, S x, \ldots, S^{N-1} x\right) \quad \text { for all } x \in \Omega_{0} .
$$




\section{Proof of Theorem 1.6: Uniqueness}

We now deal with part (5) of Theorem 1.6. $H_{\infty}$ will denote an optimal concave-convex $N$-sub-antisymmetric associated to the vector fields $u_{1}, \ldots, u_{N-1}$ obtained via the above variational procedure.

Lemma 5.1 Assume that the vector fields $u_{1}, \ldots, u_{N-1}$ from $\Omega$ to $\mathbb{R}^{d}$ are such that

$$
\left(u_{1}(x), \ldots, u_{N-1}(x)\right) \in \partial_{2, \ldots, N} H_{1}\left(x, S x, \ldots, S^{N-1} x\right) \quad \text { a.e. } x \in \Omega,
$$

for some concave-convex $N$-sub-antisymmetric Hamiltonian $H_{1}$ and some $N$-involution $S$, then $\left(H_{1}, S\right)$ is an "extremal pair", meaning that the infimum $\left(\mathrm{DK}_{\mathrm{sym}}^{2}\right)$ is attained at $H_{1}$ and the supremum $\left(\mathrm{MK}_{\text {cyc }}\right)$ is attained at $S$. Moreover, we have

$$
\left(u_{1}(x), \ldots, u_{N-1}(x)\right) \in \partial_{2, \ldots, N} H_{\infty}\left(x, S x, \ldots, S^{N-1} x\right) \quad \text { a.e. } x \in \Omega
$$

where $H_{\infty}$ is the optimal Hamiltonian constructed in Theorem 1.6 .

Proof. Let $L$ be the Fenchel-Legendre dual of $H_{1}$ with respect to the last $N-1$ variable. We have that $L_{H_{1}} \leq L$ on $\left(\mathbb{R}^{d}\right)^{N-1} \times \Omega$. It follows that

$$
\begin{aligned}
\sum_{i=1}^{N-1}\left\langle u_{i}(x), S^{i}(x)\right\rangle & \leq L_{H_{1}}\left(x, u_{1}(x), \ldots, u_{N-1}(x)\right)+H_{1}\left(x, S x, \ldots, S^{N-1} x\right) \\
& \leq L\left(x, u_{1}(x), \ldots, u_{N-1}(x)\right)+H_{1}\left(x, S x, \ldots, S^{N-1} x\right) \\
& =\sum_{i=1}^{N-1}\left\langle u_{i}(x), S^{i}(x)\right\rangle
\end{aligned}
$$

from which we deduce that

$$
\sum_{i=1}^{N-1}\left\langle u_{i}(x), S^{i}(x)\right\rangle=L_{H_{1}}\left(x, u_{1}(x), \ldots, u_{N-1}(x)\right)+H_{1}\left(x, S x, \ldots, S^{N-1} x\right),
$$

and

$$
\int_{\Omega} \sum_{i=1}^{N-1}\left\langle u_{i}(x), S^{i}(x)\right\rangle d \mu=\int_{\Omega} L_{H_{1}}\left(x, u_{1}(x), \ldots, u_{N-1}(x)\right) d \mu+\int_{\Omega} H_{1}\left(x, S x, \ldots, S^{N-1} x\right) d \mu .
$$

Use now the optimality of $H_{1}$ compared to $H_{1}-r H_{1}$ for $-1<r<1$ (Indeed, the above equality will be an inequality when $H_{1}$ is replaced by $H_{1}-r H_{1}$ for $r \neq 0$ ) and the same argument as in the proof of the existence part in Theorem 1.6 for $H_{\infty}$ to obtain that $\int_{\Omega} H_{1}\left(x, S x, \ldots, S^{N-1} x\right) d \mu=0$. On the other hand, we have

$$
\int_{\Omega} \sum_{i=1}^{N-1}\left\langle u_{i}(x), S^{i}(x)\right\rangle d \mu \leq \mathrm{MK}_{c y c}=\mathrm{DK}_{\mathrm{sym}}^{2} \leq \int_{\Omega} L_{H_{1}}\left(x, u_{1}(x), \ldots, u_{N-1}(x)\right) d \mu,
$$

which yields

$$
\int_{\Omega} \sum_{i=1}^{N-1}\left\langle u_{i}(x), S^{i}(x)\right\rangle d \mu=\mathrm{MK}_{c y c}=\mathrm{DK}_{\mathrm{sym}}^{2}=\int_{\Omega} L_{H_{1}}\left(x, u_{1}(x), \ldots, u_{N-1}(x)\right) d \mu .
$$

Now we can show that $u_{i}(x) \in \partial_{i+1} H_{\infty}\left(x, S x, \ldots, S^{N-1} x\right)$ a.e. In fact,

$$
\begin{aligned}
\int_{\Omega} \sum_{i=1}^{N-1}\left\langle u_{i}(x), S^{i}(x)\right\rangle d \mu & =\int_{\Omega} L_{H_{1}}\left(x, u_{1}(x), \ldots, u_{N-1}(x)\right) d \mu \\
& =\operatorname{DK}_{\mathrm{sym}}^{2}=\int_{\Omega} L_{H_{\infty}}\left(x, u_{1}(x), \ldots, u_{N-1}(x)\right) d \mu \\
& \geq \int_{\Omega} L_{\infty}\left(x, u_{1}(x), \ldots, u_{N-1}(x)\right) d \mu+\int_{\Omega} H_{\infty}\left(x, S x, \ldots, S^{N-1} x\right) d \mu \\
& \geq \int_{\Omega} \sum_{i=1}^{N-1}\left\langle u_{i}(x), S^{i}(x)\right\rangle d \mu,
\end{aligned}
$$


which implies that

$$
\sum_{i=1}^{N-1}\left\langle u_{i}(x), S^{i}(x)\right\rangle=L_{H_{\infty}}\left(x, u_{1}(x), \ldots, u_{N-1}(x)\right)+H_{\infty}\left(x, S x, \ldots, S^{N-1} x\right) \text { a.e. on } \Omega,
$$

and hence the desired result.

Lemma 5.2 Suppose $S$ is a measure preserving $N$-involution and $u_{i}(x)=\nabla_{i+1} H_{\infty}\left(x, S x, \ldots, S^{N-1}\right)$ a.e. for $i=1, \ldots, N-1$. Then

$$
\nabla_{1} H_{\infty}\left(x, S x, \ldots, S^{N-1} x\right)=-\sum_{i=1}^{N-1} u_{i}\left(S^{N-i} x\right) \quad \text { a.e. } x \in \Omega .
$$

Proof. Let $u \in \mathbb{R}^{d}$ and let $|t|$ be small. Note that

$$
\int_{\Omega} \sum_{i=1}^{N} H_{\infty}\left(\sigma^{N+1-i}\left(x, S x, \ldots, S^{N-1} x\right)\right) d \mu=N \int_{\Omega} H_{\infty}\left(x, S x, \ldots, S^{N-1} x\right) d \mu=0 .
$$

Since $\sum_{i=1}^{N} H_{\infty}\left(\sigma^{N+1-i}\left(x, S x, \ldots, S^{N-1}(x)\right)\right) \leq 0$, it follows that

$$
\sum_{i=1}^{N} H_{\infty}\left(\sigma^{N+1-i}\left(x, S x, \ldots, S^{N-1} x\right)\right)=0 \quad \text { a.e. } x \in \Omega .
$$

Note that $H_{\infty}$ is $N$-sub-antisymmetric and therefore

$$
\sum_{i=1}^{N} H_{\infty}\left(\sigma^{N+1-i}\left(x+t u, S x, \ldots, S^{N-1} x\right)\right) \leq 0=\sum_{i=1}^{N} H_{\infty}\left(\sigma^{N+1-i}\left(x, S x, \ldots, S^{N-1} x\right)\right) .
$$

Assuming $x$ is a point where $\nabla_{i} H_{\infty}\left(\sigma^{N+1-i}\left(x+t u, S x, \ldots, S^{N-1} x\right)\right)$ exists for all $i=1, \ldots, N$, then

$$
\sum_{i=1}^{N} \nabla_{i} H_{\infty}\left(\sigma^{N+1-i}\left(x, S x, \ldots, S^{N-1} x\right)\right)=0 .
$$

Since $u_{i}(x)=\nabla_{i+1} H_{\infty}\left(x, S x, . ., S^{N-1} x\right)$ and $S^{N}=I$ a.e., we have for $i=2,3, \ldots, N$,

$$
u_{i-1}\left(S^{N+1-i} x\right)=\nabla_{i} H_{\infty}\left(\sigma^{N+1-i}\left(x, S x, \ldots, S^{N-1} x\right)\right) .
$$

Therefore,

$$
\sum_{i=1}^{N-1} u_{i}\left(S^{N-i} x\right)+\nabla_{1} H_{\infty}\left(x, S x, \ldots, S^{N-1} x\right)=0
$$

Proposition 5.1 Let $u_{1}, \ldots, u_{N-1}$ be vector fields in $W_{\text {loc }}^{1,1}(\Omega)$ such that for any two families of points $x_{1}, \ldots, x_{N}$ and $y_{1}, \ldots, y_{N}$ in $\Omega$, the function

$$
x \rightarrow \sum_{i=1}^{N-1}\left\langle u_{i}(x), y_{i}-x_{i}\right\rangle+\sum_{i=1}^{N-1}\left\langle u_{i}\left(y_{N-i}\right)-u_{i}\left(x_{N-i}\right), x\right\rangle
$$

has no critical point unless when $x_{1}=y_{1}$. Then, there is a unique measure preserving $N$-involution $S$ on $\Omega$ that satisfies (26) for some concave-convex $N$-sub-antisymmetric Hamiltonian $H$.

Proof. Suppose $S_{1}, S_{2}$ are two measure preserving $N$-involutions on $\Omega$ and $H_{1}$ and $H_{2}$ are two concaveconvex $N$-sub-antisymmetric Hamiltonian on $\Omega \times \Omega^{N-1}$ such that for $j=1,2$, we have

$$
u_{i}(x)=\nabla_{i} H_{j}\left(x, S_{j}^{1} x, \ldots, S_{j}^{N-1}\right) \quad i=1, \ldots, N-1 .
$$


Note first that Lemma 5.1 gives that

$$
u_{i}(x)=\nabla_{i} H_{\infty}\left(x, S_{j}^{1} x, \ldots, S_{j}^{N-1}\right) .
$$

From Lemma 5.2, we have that

$$
-\sum_{i=1}^{N-1} u_{i}\left(S_{j}^{N-i} x\right)=\nabla_{1} H_{\infty}\left(x, S_{j}^{1} x, \ldots, S_{j}^{N-1}\right) .
$$

Note that the function $x \rightarrow L_{\infty}\left(x, u_{1}, \ldots, u_{N-1}(x)\right)$ is locally Lipschitz and therefore is differentiable on a subset $\Omega_{0}$ of full measure. We now show that $S_{1}=S_{2}$ on $\Omega_{0}$.

Indeed, for any $x \in \Omega_{0}, h=0$ is a minimum for the function

$$
h \rightarrow L_{\infty}\left(x+h, u_{1}(x+h), \ldots, u_{N-1}(x+h)\right)+H_{\infty}\left(x+h, S_{j}^{1} x, \ldots, S_{j}^{N-1} x\right)-\sum_{i=1}^{N-1}\left\langle u_{i}(x+h), S_{j}^{i}(x)\right\rangle .
$$

This implies that

$$
\begin{aligned}
\nabla_{1} H_{\infty}\left(x, S_{1}^{1} x, \ldots, S_{1}^{N-1} x\right)-\sum_{i=1}^{N-1}\left\langle\nabla u_{i}(x), S_{1}^{i}(x)\right\rangle & =-\frac{d}{d h} L_{\infty}\left(x+h, u_{1}(x+h), \ldots, u_{N-1}(x+h)\right)_{h=0} \\
& =\nabla_{1} H_{\infty}\left(x, S_{2}^{1} x, \ldots, S_{2}^{N-1} x\right)-\sum_{i=1}^{N-1}\left\langle\nabla u_{i}(x), S_{2}^{i}(x)\right\rangle .
\end{aligned}
$$

This yields that

$$
\begin{aligned}
\sum_{i=1}^{N-1}\left\langle\nabla u_{i}(x), S_{2}^{i}(x)-S_{1}^{i}(x)\right\rangle & =\nabla_{1} H_{\infty}\left(x, S_{2}^{1} x, \ldots, S_{2}^{N-1} x\right)-\nabla_{1} H_{\infty}\left(x, S_{1}^{1} x, \ldots, S_{1}^{N-1} x\right) \\
& =\sum_{i=1}^{N-1}\left(u_{i}\left(S_{1}^{N-i}(x)\right)-u_{i}\left(S_{2}^{N-i}(x)\right) .\right.
\end{aligned}
$$

The hypothesis then implies that $S_{1}(x)=S_{2}(x)$, and $S$ is therefore unique.

In order to find examples of families of vector fields satisfying the above sufficient condition for uniqueness, we look again at $N$-monotone vector fields. For that we introduce the following notion.

Definition 5.3 Say that a family of vector fields $\left(u_{1}, u_{2}, \ldots, u_{N-1}\right)$ on $\Omega$ is strictly jointly $N$-monotone if

$$
\sum_{i=1}^{N} \sum_{l=1}^{N-1}\left\langle u_{l}\left(x_{i}\right), x_{i}-x_{i+l}\right\rangle>0
$$

for every cycle $x_{1}, \ldots, x_{2 N-1}$ of points in $\Omega$ such that $x_{N+l}=x_{l}$ for $1 \leq l \leq N-1$, and $x_{1} \neq x_{2}$.

Note that for $N=2$, this property means that the vector field $u_{1}$ is strictly 2-monotone, that is,

$$
\left\langle u_{1}(y)-u_{1}(x), y-x\right\rangle>0 \text { for all } y, x \in \Omega \text { with } x \neq y .
$$

In this case, it is easy to see that if $u_{1}$ is differentiable, then strict monotonicity implies the sufficient condition for uniqueness mentioned in Proposition 5.1. Indeed, let $u \in \mathbb{R}^{d}$ and $x \in \Omega$. By taking $y=x+t u$ in (61) and letting $t \rightarrow 0^{+}$we obtain $\left\langle\nabla u_{1}(x) u, u\right\rangle \geq 0$.

Assume now that the function $x \rightarrow\left\langle u_{1}(x), y_{1}-x_{1}\right\rangle+\left\langle u_{1}\left(y_{1}\right)-u_{1}\left(x_{1}\right), x\right\rangle$ has a critical point and that $y_{1} \neq x_{1}$. It follows that

$$
\left\langle\nabla u_{1}(x)\left(y_{1}-x_{1}\right), y_{1}-x_{1}\right\rangle+\left\langle u_{1}\left(y_{1}\right)-u_{1}\left(x_{1}\right), y_{1}-x_{1}\right\rangle=0 .
$$


Since the first term is non-negative and the second one is strictly positive, this leads to a contradiction.

One can however, establish directly the following uniqueness result for strictly jointly $N$-monotone families for $N \geq 2$, even without the differentiability assumption on $u_{1}, \ldots, u_{N-1}$. This is because we already know from the result of Galichon-Ghoussoub [6] mentioned in the introduction that $S_{1}(x)=x$ is one of the possible $N$-involution measure preserving maps in the representation of $\left(u_{1}, \ldots, u_{N-1}\right)$.

Proposition 5.2 Assume $u_{1}, \ldots, u_{N-1}$ is a strictly jointly $N$-monotone family of bounded vector fields on $\Omega$. Then, $S=I$ is the only measure preserving $N$-involution $S$ on $\Omega$ that satisfies (26) for some concave-convex $N$-sub-antisymmetric Hamiltonian $H$.

Proof. Assume $S$ is another measure preserving $N$-involution in the decomposition. Let $x_{i}=S^{i} x$ for $i=1,2, \ldots, N$ and note that $x_{N}=x$. It follows from (60) that

$$
\sum_{i=0}^{N-1} \sum_{l=1}^{N-1}\left\langle u_{l}\left(S^{i} x\right), S^{i} x-S^{i+l} x\right\rangle \geq 0 .
$$

Integrating the above expression over $\Omega$ implies that

$$
\begin{aligned}
0 & \leq \int_{\Omega} \sum_{i=0}^{N-1} \sum_{l=1}^{N-1}\left\langle u_{l}\left(S^{i} x\right), S^{i} x-S^{i+l} x\right\rangle d \mu \\
& =\sum_{i=0}^{N-1} \sum_{l=1}^{N-1} \int_{\Omega}\left\langle u_{l}\left(S^{i} x\right), S^{i} x\right\rangle d \mu-\sum_{i=0}^{N-1} \sum_{l=1}^{N-1} \int_{\Omega}\left\langle u_{l}\left(S^{i} x\right), S^{i+l} x\right\rangle d \mu \\
& =\sum_{i=0}^{N-1} \sum_{l=1}^{N-1} \int_{\Omega}\left\langle u_{l}(x), x\right\rangle d \mu-\sum_{i=0}^{N-1} \sum_{l=1}^{N-1} \int_{\Omega}\left\langle u_{l}(x), S^{l} x\right\rangle d \mu \\
& =N \sum_{l=1}^{N-1} \int_{\Omega}\left\langle u_{l}(x), x\right\rangle d \mu-N \sum_{l=1}^{N-1} \int_{\Omega}\left\langle u_{l}(x), S^{l} x\right\rangle d \mu \\
& =N \int_{\Omega} L_{\infty}\left(x, u_{1}(x), \ldots, u_{N-1}(x)\right) d \mu-N \int_{\Omega} L_{\infty}\left(x, u_{1}(x), \ldots, u_{N-1}(x)\right) d \mu \\
& =0
\end{aligned}
$$

The latter identity is because both terms correspond to the optimal value $\left(\mathrm{MK}_{c y c}\right)$. Since the integrand in the first line of the above expression is nonnegative we obtain

$$
\sum_{i=0}^{N-1} \sum_{l=1}^{N-1}\left\langle u_{l}\left(S^{i} x\right), S^{i} x-S^{i+l} x\right\rangle=0, \quad \text { a.e. } x \in \Omega,
$$

and therefore $S x=x$.

\section{Proof of Theorem 1.7}

The question here is what happens when some of the vector fields $u_{i}$ are identically zero. Let us illustrate the situation by assuming that just one of them, say $u_{N-1} \equiv 0$. In this case, there are two scenarios:

(I) One can begin with $N-2$ vectors $u_{1}, . ., u_{N-2}$, and obtain a sub $(N-1)$-antisymmetric Hamiltonian $H$ and an $(N-1)$-involution $S$ such that $u_{i}(x) \in \partial H_{i+1}\left(x, S x, \ldots, S^{N-2} x\right)$.

(II) One can proceed as above, while considering $u_{N-1} \equiv 0$ as a vector field like the others. Note that in the proof of the main theorem we never assumed $u_{N-1} \neq 0$, except on line (57) and the preceding paragraph. However, it is easily seen that by assuming $u_{N-1} \equiv 0$, one still gets

$$
\left(u_{1}(x), \ldots, u_{N-2}(x)\right) \in \partial_{2, \ldots, N-2} H_{\infty}\left(x, S x, \ldots, S^{N-1} x\right) \quad \text { a.e. } \quad x \in \Omega .
$$


and the dependence of the Hamiltonian $H_{\infty}$ with respect to the $N$-th variable seems to be redundant. In this case $H_{\infty}$ can be chosen to be an $N$-antisymmetric Hamiltonian, which depends on only $N-1$ variables. This is because $H_{r e g}^{1}\left(x_{1}, \ldots, x_{N}\right)=H_{L_{H}^{* *}}\left(x_{1}, \ldots, x_{N-1}, x_{N}\right)$ can be replaced by

$$
H_{r e g}^{0}\left(x_{1}, \ldots, x_{N-1}, x_{N}\right):=F_{0}\left(x_{1}, \ldots, x_{N-1}\right),
$$

where

$$
F_{0}\left(x_{1},, \ldots, x_{N-1}\right)=\sup _{p_{2}, \ldots, p_{N-1} \in B_{R}}\left\{\sum_{i=2}^{N-1}\left\langle p_{i}, x_{i}\right\rangle-L_{H}^{* *}\left(x_{1}, p_{2}, \ldots, p_{N-1}, 0\right)\right\} .
$$

Indeed, it follows from (46) that for all $x_{i} \in \Omega$ and $p_{j}^{i} \in \mathbb{R}^{d}$ the following inequality holds

$$
\sum_{i=1}^{N}\left\{\sum_{j=1, j \neq i}^{N}\left\langle p_{j}^{i}, x_{j}\right\rangle-L_{H}^{* *}\left(R^{i-1}\left(p_{1}^{i}, \ldots, p_{i-1}^{i}, x_{i}, p_{i+1}^{i}, \ldots, p_{N}^{i}\right)\right)\right\} \leq 0 .
$$

In the above expression, set $p_{i-1}^{i}=p_{N}^{1}=0$ for $i>1$. By taking sup over all non-zero $p_{i}^{j} \in B_{R}$ we obtain

$$
\sum_{i=1}^{N} H_{r e g}^{0}\left(\sigma^{i-1}\left(x_{1}, \ldots, x_{N}\right)\right) \leq 0 .
$$

This proves that $H_{\text {reg }}^{0}$ is $N$-sub-antisymmetric. By defining

$$
H_{r e g}^{2}(\mathbf{x})=\frac{(N-1) H_{r e g}^{0}(\mathbf{x})-\sum_{i=1}^{N-1} H_{r e g}^{0}\left(\sigma^{i}(\mathbf{x})\right)}{N},
$$

and using a similar argument as in the proof of Proposition 3.1. one can also obtain that $L_{H_{r e g}^{2}} \leq L_{H_{r e g}^{0}} \leq L_{H}$ on $\bar{\Omega} \times\left(B_{R}\right)^{N-1}$. This together with ([62) and (63) imply that the Hamiltonian $H_{\infty}$ obtained variationally in Theorem 1.6 can be chosen to be independent with respect to the last variable.

Similarly, one can show that if more than one vector fields is zero, then the dependence of $H_{\infty}$ on the corresponding variables can be dropped.

Suppose now that $u_{2}=\ldots=u_{N-1}=0$. In this case $H_{\infty}$ is just a function of two variables, i.e. $H\left(x_{1}, x_{2}, \ldots, x_{N}\right)=F\left(x_{1}, x_{2}\right)$ for some Lipschitz function $F$, which is concave with respect to the first variable and convex with respect to the second one. Therefore $u_{1}(x) \in \partial_{2} F(x, S x)$ for some measure preserving $N$-involution. In this case, the sub-N-antisymmetry of $H_{\infty}$ translates into

$$
\sum_{i=1}^{N} F\left(x_{i+1}, x_{i}\right) \leq 0 \text { for all } x_{1}, \ldots x_{N} \in \Omega \text { with } x_{1}=x_{N+1} .
$$

\section{Appendix}

Theorem 7.1 Consider bounded vector fields $\left(u_{i}\right)_{i=1}^{N-1}$ on $\Omega$ such that for $i=1,2, \ldots, N-1$,

$$
u_{i}(x) \in \partial_{i+1} H\left(x, S x, \ldots, S^{N-1} x\right) \text { a.e. } \Omega,
$$

where $S: \bar{\Omega} \rightarrow \bar{\Omega}$ is a measure preserving $N$-involution, and $H: \mathbb{R}^{d} \times\left(\mathbb{R}^{d}\right)^{N-1}$ is a Lipschitz function satisfying the following properties:

1. $H(., X)$ is concave for every $X \in\left(\mathbb{R}^{d}\right)^{N-1}$, and $H(x,$.$) is convex for all x \in \mathbb{R}^{d}$.

2. $H$ is $N$-sub-antisymmetric on $(\bar{\Omega})^{N}$.

3. $\int_{\Omega} H\left(x, S x, \ldots, S^{N-1} x\right) d \mu=0$.

If either $\left.S \in W_{\text {loc }}^{1,1}(\Omega)\right)$ or $u_{i} \in W_{\text {loc }}^{1,1}(\Omega)$ for $i=1,2, \ldots, N-1$, then there exists a full measure subset $\Omega_{0}$ of $\Omega$ such that $\nabla_{i} H\left(x, S x, \ldots, S^{N-1} x\right)$ exists for all $x \in \Omega_{0}$. 
First recall the following standard lemma.

Lemma 7.2 Let $f: \mathbb{R}^{n} \rightarrow(-\infty,+\infty]$ be a proper convex function and let $x$ be a point where $f$ is finite. The following statements hold:

1. For each $v \in \mathbb{R}^{n}$, the difference quotient in the definition of $D f(x) v$ is a non-decreasing function of $\lambda>0$, so that $\operatorname{Df}(x) v$ exists and

$$
D f(x) v=\inf _{\lambda>0} \frac{f(x+\lambda v)-f(x)}{\lambda}
$$

2. the function $v \rightarrow D f(x) v$ is a positively homogeneous convex function of $v$ with

$$
D f(x) u+D f(x)(-v) \geq 0 \quad \forall v \in \mathbb{R}^{n} .
$$

Lemma 7.3 For each $v \in \mathbb{R}^{d}$, we have

$$
\int_{\Omega} D_{1} H\left(x, S x, S^{2} x, \ldots, S^{N-1} x\right)(v) d \mu+\int_{Q} D_{1} H\left(x, S x, S^{2} x, \ldots, S^{N-1} x\right)(-v) d \mu=0 .
$$

Proof. Let $t>0$ and define

$$
\begin{aligned}
I^{1}(x, v, t) & =H\left(x, S(x+t v), S^{2}(x+t v), \ldots, S^{N-1}(x+t v)\right) \\
I^{2}(x, v, t) & =H\left(x+t v, S x, S^{2} x, \ldots, S^{N-1} x\right)
\end{aligned}
$$

Let $g \in C_{c}^{\infty}(\Omega)$ be a non-negative function. By a simple change of variables, we have for $t>0$ small enough,

$$
\begin{gathered}
\int_{\Omega} \frac{I^{1}(x, v, t) g(x)+I^{1}(x,-v, t) g(x)-2 I^{1}(x, 0,0) g(x)}{t} d \mu= \\
\int_{\Omega} \frac{I^{2}(x,-v, t) g(x-t v)+I^{2}(x, v, t) g(x+t v)-2 I^{1}(x, 0,0) g(x)}{t} d \mu .
\end{gathered}
$$

The limit of the right hand side of the above expression exists as $t \rightarrow 0^{+}$and

$$
\begin{array}{r}
\lim _{t \rightarrow 0^{+}} \int_{\Omega} \frac{I^{2}(x,-v, t) g(x-t v)+I^{2}(x, v, t) g(x+t v)-2 I^{1}(x, 0,0) g(x)}{t} d \mu= \\
\int_{\Omega}\left[D_{1} H\left(x, S x, S^{2} x, \ldots, S^{N-1} x\right)(v)+D_{1} H\left(x, S x, S^{2} x, \ldots, S^{N-1} x\right)(-v)\right] g(x) d \mu \leq 0,
\end{array}
$$

where the last inequality is due to the concavity of $H$ with respect to the first variable. We shall now prove that the limit of the left hand side of (66) is non-negative as $t \rightarrow 0^{+}$. It follows from the convexity of $H$ with respect to the last $N-1$ variable together with $u_{i}(x) \in \partial_{i+1} H\left(x, S x, \ldots, S^{N-1} x\right)$ that

$$
\begin{gathered}
\int_{\Omega} \frac{I^{1}(x, v, t) g(x)+I^{1}(x,-v, t) g(x)-2 I^{1}(x, 0,0) g(x)}{t} d \mu \geq \\
\frac{1}{t} \int_{\Omega} \sum_{i=1}^{N-1}\left\langle u_{i}(x), S^{i}(x+t v)+S^{i}(x-t v)-2 S(x)\right\rangle g(x) d \mu .
\end{gathered}
$$

The right hand side of the above expression goes to zero, as $t \rightarrow 0$, provided either $S \in W_{l o c}^{1,1}(\Omega)$ or $u_{i} \in W_{l o c}^{1,1}(\Omega)$ for $i=1,2, \ldots, N-1$. This together with (66) and (67) imply that

$$
\int_{\Omega}\left[D_{1} H\left(x, S x, S^{2} x, \ldots, S^{N-1} x\right)(v)+D_{1} H\left(x, S x, S^{2} x, \ldots, S^{N-1} x\right)(-v)\right] g(x) d \mu=0,
$$

from which the desired results follows. 
Lemma 7.4 For $v \in \mathbb{R}^{d}$, define $G_{i}(v)=\int_{\Omega} D_{i} H\left(x, S x, \ldots, S^{N-1} x\right)(v) d \mu$. Then

$$
\sum_{i=1}^{N}\left[G_{i}(v)+G_{i}(-v)\right] \leq 0 .
$$

Proof. Define $f_{i}(t, x, v)=H\left(\sigma^{N+1-i}\left(x+t v, S x, \ldots, S^{N-1} x\right)\right)$. Note that

$$
t \rightarrow \frac{f_{i}(t, x, v)+f_{i}(t, x,-v)-2 f_{i}(0, x, v)}{t}
$$

is monotone and does not change sign. It follows from the monotone convergence theorem that

$$
\begin{array}{r}
\lim _{t \rightarrow 0^{+}} \int_{\Omega} \frac{f_{i}(t, x, v)+f_{i}(t, x,-v)-2 f_{i}(0, x, v)}{t} d \mu=\int_{\Omega} \lim _{t \rightarrow 0^{+}} \frac{f_{i}(t, x, v)+f_{i}(t, x,-v)-2 f_{i}(0, x, v)}{t} d \mu \\
=\int_{\Omega}\left[D_{i} H\left(\sigma^{N+1-i}\left(x, S x, \ldots, S^{N-1} x\right)\right)(v)+D_{i} H\left(\sigma^{N+1-i}\left(x, S x, \ldots, S^{N-1} x\right)\right)(v-)\right] d \mu \\
=\int_{\Omega}\left[D_{i} H\left(x, S x, \ldots, S^{N-1} x\right)(v)+D_{i} H\left(x, S x, \ldots, S^{N-1} x\right)(-v)\right] d \mu=G_{i}(v)+G_{i}(-v) .
\end{array}
$$

Let $\chi_{\Omega}(t, x)$ be a function that is one when both $x+t v, x-t v \in \Omega$ and zero otherwise. It follows from the dominated convergence theorem that

$$
\begin{aligned}
G_{i}(v)+G_{i}(-v) & =\int_{\Omega} \lim _{t \rightarrow 0^{+}} \frac{f_{i}(t, x, v)+f_{i}(t, x,-v)-2 f_{i}(0, x, v)}{t} \chi_{\Omega}(t, x) d \mu \\
& =\lim _{t \rightarrow 0^{+}} \int_{\Omega} \frac{f_{i}(t, x, v)+f_{i}(t, x,-v)-2 f_{i}(0, x, v)}{t} \chi_{\Omega}(t, x) d \mu .
\end{aligned}
$$

Let $f(t, x, v)=\sum_{i=1}^{N} f_{i}(t, x, v)$. Note that for each $x \in \Omega$ one has $f(t, x, v)=\sum_{i=1}^{N} f_{i}(t, x, v) \leq 0$ for $t$ small enough such that $x+t v \in \Omega$. Similarly $f(t, x,-v) \leq 0$ for $x-t v \in \Omega$. One also has that $\int_{\Omega} f(0, x, v) d \mu=0$. It follows that

$$
\begin{aligned}
\sum_{i=1}^{N}\left[G_{i}(v)+G_{i}(-v)\right] & =\int_{\Omega} \lim _{t \rightarrow 0^{+}} \frac{f(t, x, v)+f(t, x,-v)-2 f(0, x, v)}{t} \chi_{\Omega}(t, x) d \mu \\
& =\lim _{t \rightarrow 0^{+}} \int_{\Omega} \frac{f(t, x, v)+f(t, x,-v)-2 f(0, x, v)}{t} \chi_{\Omega}(t, x) d \mu \\
& =\lim _{t \rightarrow 0^{+}} \int_{\Omega} \frac{f(t, x, v)+f(t, x, v)}{t} \chi_{\Omega}(t, x) d \mu \leq 0 .
\end{aligned}
$$

Proof of Theorem [7.1. Fom Lemma 7.3 and 7.4 we have for each $v \in \mathbb{R}^{d}$ and $i=1,2, \ldots, N$

$$
\int_{\Omega}\left[D_{i} H\left(x, S x, S^{2} x, \ldots, S^{N-1} x\right)(v)+D_{i} H\left(x, S x, S^{2} x, \ldots, S^{N-1} x\right)(-v)\right] d \mu=0 .
$$

Since the integrand does not change sign, it has to be zero almost everywhere. Now choose $\left\{v_{k}\right\}_{k=1}^{\infty}$ to be a countable dense subset of $\mathbb{R}^{d}$. Set

$$
A_{k}=\left\{x \in \Omega ; D_{i} H\left(x, S x, S^{2} x, \ldots, S^{N-1} x\right)\left(v_{k}\right)+D_{i} H\left(x, S x, S^{2} x, \ldots, S^{N-1} x\right)\left(-v_{k}\right)=0, \quad 1 \leq i \leq N\right\}
$$

It follows from (68) that $\Omega \backslash A_{k}$ is a null set and $\Omega_{0}=\cap_{k} A_{k}$ is a full measure subset of $\Omega$ such that $\nabla_{i} H\left(x, S x, S^{2} x, \ldots, S^{N-1} x\right)$ exists for all $x \in \Omega_{0}$.

\section{References}

[1] Y. Brenier, Polar factorization and monotone rearrangement of vector-valued functions, Comm. Pure Appl. Math. 44 (1991), 375-417. 
[2] G. Buttazzo, L. De Pascale, P. Gori-Giorgi, Optimal-transport formulation of electronic densityfunctional theory, Physical Review A (2012) pp. 062502-1-11

[3] M. Colombo, S. Di Marino, Equality between Monge and Kantorovich multimarginal problems with Coulomb cost, Preprint, April 11, 2013

[4] C. Cotar, G. Friesecke, C. Klppelberg, Density functional theory and optimal transportation with Coulomb cost, Commun. Pure Appl. Math. 66 (2013) 54899

[5] C. Cotar, G. Friesecke, B. Pass, Infinite-body optimal transport with Coulomb cost, arXiv:1307.6540v1 (24 July 2013)

[6] A. Galichon, N. Ghoussoub, Variational representations for $N$-cyclically monotone vector fields, arXiv:1207.2408v2 [math.OC] (2012)

[7] W. Gangbo, An elementay proof of the polar factorization of vector-valued functions, Arch. Rat. Math. Analysis 128, No.5, (1994) 381-399.

[8] W. Gangbo, A. Święch, Optimal maps for the multidimensional Monge-Kantorovich problem Comm. Pure Appl. Math., 51(1) (1998) 23-45.

[9] N. Ghoussoub, Selfdual partial differential systems and their variational principles, Springer Monograph in Mathematics, Springer-Verlag (2008), 356 p.

[10] N. Ghoussoub, B. Maurey, Remarks on multidimensional symmetric Monge-Kantorovich problems, Discrete and Continuous Dynamical Systems-A, special issue on "Optimal Transport and Applications" (2012)

[11] N. Ghoussoub, A. Moameni, A Self-dual Polar Factorization for Vector Fields, Comm. Pure. Applied. Math., Vol 66, Issue 6 (2013) p. 905-933

[12] E. Krauss, A representation of arbitrary maximal monotone operators via subgradients of skewsymmetric saddle functions, Nonlinear Anal. 9 (1985), no. 12, 1381-1399.

[13] B. Pass, PhD thesis, University of Toronto, 2011.

[14] Pass, B., Uniqueness and Monge solutions in the multimarginal optimal transportation problem, SIAM Journal on Mathematical Analysis, 43(6) (2011), 2758-2775

[15] A. Plakhov, Billiards, Optimal mass transport and Problems of optimal aerodynamic resistance, Journal of Mathematical Sciences, Vol. 182, No. 2, April, 2012

[16] T. Rockafellar, Convex Analysis, 1970, Princeton University Press.

[17] C. Villani, Topics in optimal transportation, volume 58 of Graduate Studies in Mathematics. American Mathematical Society, Providence, 2003.

[18] C. Villani, Optimal transport: old and new, volume 338 of Grundlehren der mathematischen Wissenschaften. Springer, New York, 2009. 